\title{
CHANDRA OBSERVATIONS OF COMET 2P/ENCKE 2003: FIRST DETECTION OF A COLLISIONALLY THIN, FAST SOLAR WIND CHARGE EXCHANGE SYSTEM
}

\author{
C. M. Lisse, ${ }^{1,2}$ D. J. Christian, ${ }^{3}$ K. Dennerl, ${ }^{4}$ S. J. Wolk,${ }^{5}$ D. Bodewits, ${ }^{6}$ R. Hoekstra, ${ }^{6}$ \\ M. R. Combi, ${ }^{7}$ T. Mäkinen, ${ }^{8}$ M. Dryer, ${ }^{9}$ C. D. Fry, ${ }^{10}$ And H. Weaver ${ }^{1}$ \\ Received 2005 May 20; accepted 2005 August 24
}

\begin{abstract}
We report the results of $15 \mathrm{hr}$ of Chandra observations of comet 2P/Encke 2003 on November 24. X-ray emission from comet Encke was resolved on scales of 500-40,000 km, with unusual morphology due to the presence of a low-density, collisionally thin (to charge exchange) coma. A light curve with peak-to-peak amplitude of $20 \%$ consistent with a nucleus rotational period of $11.1 \mathrm{hr}$ was found, further evidence for a collisionally thin coma. We confirm emission lines due to oxygen and neon in the $800-1000 \mathrm{eV}$ range but find very unusual oxygen and carbon line ratios in the 200-700 eV range, evidence for low-density, high effective temperature solar wind composition. We compare the X-ray spectral observation results to contemporaneous measurements of the coma and solar wind made by other means and find good evidence for the dominance of a postshock bubble of expanding solar wind plasma, moving at $600 \mathrm{~km} \mathrm{~s}^{-1}$ with charge state composition between that of the "fast" and "slow" solar winds.
\end{abstract}

Subject headings: comets: individual (2P/Encke 2003) — solar wind - X-rays: general

Online material: color figure

\section{INTRODUCTION}

Cometary X-ray emission, first discovered in 1996 and now observed in over 20 comets, has been shown to be caused by charge exchange reactions between highly charged solar wind minor ions ( $\sim 0.1 \%$ of all solar wind ions) and neutral gas species emitted by the nucleus into the cometary coma (Cravens 1997; Dennerl et al. 1997; Kharchenko \& Dalgarno 2000, 2001; Krasnopolsky et al. 2002; Lisse et al. 1999, 2001). The emission appears to be confined to the sunward hemisphere of the neutral coma, with emission-line energies characteristic of atomic cascades from hydrogen-like and helium-like $\mathrm{C}, \mathrm{N}, \mathrm{O}$, and $\mathrm{Ne}$ and a luminosity proportional to the solar wind ion flux density.

The implications of the charge exchange mechanism are manyfold and have yet to be fully explored. Variations in the emission that track spatial, temporal, and compositional changes in the solar wind should be present. The solar wind has large-scale structure relating to the very different corona environments at the equator and pole of the Sun: the low-latitude solar wind is relatively dense, slow moving $\left(v \sim 200-300 \mathrm{~km} \mathrm{~s}^{-1}\right)$, and of

\footnotetext{
${ }^{1}$ Planetary Exploration Group, Space Department, Johns Hopkins University Applied Physics Laboratory, 11100 Johns Hopkins Road, Laurel, MD 20723; carey.lisse@jhuapl.edu, hal.weaver@jhuapl.edu.

${ }_{2}$ Corresponding author.

${ }^{3}$ Queens University, Department of Pure and Applied Physics, Belfast BT7 1NN, UK; d.christian@qub.ac.uk.

${ }_{4}^{4}$ Max-Planck-Institut für extraterrestrische Physik, Postfach 1603, 85740 Garching, Germany; kod@mpe.mpg.de.

${ }_{5}$ Chandra X-Ray Observatory Center, Harvard-Smithsonian Center for Astrophysics, 60 Garden Street, Cambridge, MA 02138; swolk@cfa.harvard.edu.

${ }^{6}$ KVI Atomic Physics, Rijkuniversiteit Groningen, Zernikelaan 25, 9747 AA Groningen, Netherlands; bodewits@kvi.nl, hoekstra@kvi.nl.

7 Department of Atmospheric, Oceanic, and Space Sciences, University of Michigan, 2455 Hayward Street, Ann Arbor, MI 48109-2143; mcombi@engin .umich.edu.

${ }^{8}$ Finnish Meteorological Institute, Space Research, P.O. Box 503, FIN-00101 Helsinki, Finland; teemu.makinen@fmi.fi.

${ }^{9}$ NOAA Space Environment Center, 325 Broadway, Boulder, CO 80305; murray.dryer@noaa.gov.

10 Exploration Physics International, Inc., Suite 37-105, 6275 University Drive, Huntsville, AL 35806-1776; gfry@expi.com.
}

high temperature $\left(\sim 2 \times 10^{6} \mathrm{~K}\right)$, while the high-latitude polar wind is less dense and cooler $\left(\sim 1 \times 10^{6} \mathrm{~K}\right)$ but moving at higher average speeds $\left(v>400 \mathrm{~km} \mathrm{~s}^{-1}\right)$. These differences should cause detectable differences in the observed X-ray spectrum of the comet (Beiersdorfer et al. 2003; Bodewits et al. 2004). Limitations of the emission region to the volume of the neutral coma available to the solar wind are predicted, with evidence in the morphology and size of the emission region (Lisse \& Christian 2002; Lisse et al. 2004; Wegmann et al. 2004). Modulation of the X-ray signal due to modulations of the neutral coma density should be present for systems that are collisionally thin. The coma neutral density is modulated as actively sublimating emitting regions on the cometary nucleus rotate in and out of sunlight during the course of a comet's day.

To date, the best-observed comets in the X-ray have been close Earth approachers with high coma "activity" (i.e., rate of sublimation of volatile ices into neutral gas species in the gravitationally unbound atmosphere surrounding the nucleus). The brightest known comet target available during Chandra Cycle 5 was 2P/Encke 2003 (hereafter Encke), an old, shortperiod comet with a low production rate of dust and gas. Well observed for $\sim 60$ apparitions over the course of three centuries, the comet had a very close passage by the Earth in 2003 November-December, with a minimum Earth-comet distance of $0.26 \mathrm{AU}$ and a maximum visual brightness of $V \sim 6.4$. In 1997, at larger heliocentric and similar geocentric distance and a magnitude fainter brightness, we found Encke to be an excellent laboratory for studies of cometary X-ray emission (Lisse et al. 1999). With only broadband photometry, we were able to infer that charge exchange between highly ionized solar wind minor ions and cometary neutral gas species was the most likely emission mechanism (Lisse et al. 1999).

The highly favorable perigee passage of comet Encke in late 2003 provided an excellent opportunity to use Chandra's spatial, spectral, and temporal resolution to study cometary X-ray emission in the low neutral target density, low X-ray flux regime. The 1997 findings suggested a straightforward Chandra experiment: continuous ACIS-S observations of Encke throughout one 
nuclear revolution period of $15 \mathrm{hr}$ (as determined by Luu \& Jewitt 1990) during the time of its closest approach to Earth. Encke is the first low-activity, low-dust, short-period comet observed by Chandra, and the first comet observed in the X-ray on more than one perihelion passage, allowing us to check for reproducibility in cometary X-ray emission behavior and providing an important bridge between the Chandra and ROSAT/EUVE (Röntgensatellit/ Extreme Ultraviolet Explorer) comet databases.

In this paper, we report the results of our observations and compare the emission morphology and extracted X-ray spectra in the $200-1000 \mathrm{eV}$ range to current models. In $\S 2$ we describe the details of the instruments used and our data analysis techniques. In $\S 3$ we present the observational results, placing them in the context of previous cometary X-ray observations. In $\S 4$ we compare the observations to contemporaneous measurements of the coma and solar wind made by other means and argue for the presence of a low-density, collisionally thin (to charge exchange) coma and a fast moderate-density solar wind as a plausible physical model explaining our results. In $\S 5$ we list our observational and model-derived conclusions for X-ray emission from Encke in 2003.

\section{INSTRUMENTAL}

Chandra.-Chandra observations of comet Encke were obtained on 2003 November 24 in 15 hr (54 ks) of continuous pointing, using the ACIS-S CCD spectral array, which combines the abilities to image X-rays with a plate scale of $0.5 \mathrm{pixel}^{-1}$ and to produce accurate, moderate-resolution spectra $\left(\Delta E \sim 110 \mathrm{eV}\right.$ FWHM, $\left.\Delta E_{\text {Gaussian }}=49 \mathrm{eV}\right)$ from 0.30 to $2.0 \mathrm{keV}$. During the Chandra observation on 2003 November 24, Encke was approximately $0.88 \mathrm{AU}$ from the Sun and $0.27 \mathrm{AU}$ from the Earth, with a total visual magnitude of $V \sim 6.5$, a total luminosity of $L_{\text {optical }} \sim 2 \times 10^{19} \mathrm{ergs} \mathrm{s}^{-1}$, and an estimated $Q_{\text {gas }}$ of $\sim 2 \times 10^{28} \mathrm{~mol} \mathrm{~s}^{-1}$.

We obtained 30 pointed Chandra observations of Encke on 2003 November 24 from 04:30 to 18:30 UT. The method of Chandra observation was kept simple: the ACIS-S array was pointed on the sky $2^{\prime}$ in front of the comet along the direction of its apparent motion, and the comet was allowed to move through the field of view. No active guiding on the comet was attempted. Chandra observations return a list of time-tagged detections of individual photon pulse heights and spatial locations. Chandra was able to follow the comet's nonsidereal motion using multiple pointings, and the target was centered in the back-illuminated CCD chip S3, the most sensitive CCD for energies below $1 \mathrm{keV}$. No obvious bright background sources were detected in our data, other than the comet.

Using the sso_freeze algorithm, part of the Chandra Interactive Analysis of Observations (CIAO) software, we constructed a comet image remapped onto a coordinate system moving with the comet. The resulting effective field of view of the observations in the cometocentric coordinate system is $10^{\prime} \times 10^{\prime}$. Background removal was performed in two different ways: (1) counting all photons within a circular aperture of $550^{\prime \prime}$ and subtracting the signal measured in an annular radius of $200^{\prime \prime}$ outside this using the CIAO reduction package, and (2) creating a signal versus aperture width curve using IDL, after removing a background estimated from a "frame" 20 pixels wide around the edge of the field of view in cometocentric coordinates. The two methods agree to within $20 \%$ in the amount of background counts per pixel. A total of 6500 photons attributable to the comet were found after spatial background removal using XSPEC fitting routines (Arnaud 1996), for an average count rate over the 54 ks of observations of 0.12 counts s ${ }^{-1}$. To create light curves, a circular source aperture of 3.7 radius was chosen in CIAO, and several background apertures were extracted $>4^{\prime}$ away from the comet's diffuse emission and toward the outer edge of the chip. Spectra were extracted in an identical fashion, and the effect of energy-dependent instrument sensitivity was removed using the latest effective areas given in the Chandra on-orbit measured instrument response matrices. Upon examination of the extracted spectra, only photons of energy $0.20-1.0 \mathrm{keV}$ were found to be statistically significant against the sky and instrumental backgrounds.

SOHO and ACE.-SOHO (Solar and Heliospheric Observatory) measurements of the solar wind proton flux, solar soft $\mathrm{X}$-ray flux, and solar wind magnetic field were obtained using the CELIAS instrument. ${ }^{11} A C E$ (Advanced Composition Explorer) ULEIS ultrasoft ion spectroscopy data for $\mathrm{H}, \mathrm{He}^{4}, \mathrm{C}, \mathrm{O}$, $\mathrm{Ne}$, and Fe were also used. ${ }^{12}$ Our observations of Encke using the ACIS-S camera and of the solar-terrestrial environment using $S O H O$, Wind, and $A C E$ were performed in the same manner as used to observe comets C/1999 S4 (LINEAR) (Lisse et al. 2001) and C/McNaught-Hartley 1999 T1 (Krasnopolsky et al. 2002). We refer the reader to these papers for detailed descriptions of the instruments used and the corrections applied to the data.

\section{OBSERVATIONS}

Morphology.-The emission morphology (Fig. 1a) is decidedly non-crescent shaped, unlike the overwhelming majority of the $20+$ comets observed in the X-ray to date (Lisse et al. 2004 ). At the $104^{\circ}$ phase angle of our observations, the hemispherical emission geometry consistent with an extended cometary atmosphere encountering the solar wind and fully depleting the population of minor ions in charge exchange interactions would appear as a crescent-shaped region in projection (Wegmann et al. 2004). The morphology of the emission region most closely matches the morphology of the optical coma (Figs. $1 b$ and $1 c$ ), which is relatively faint and dominated by a sunward "fan" composed of two bright jets. For this to be the case, the limiting constituent in the two-body charge exchange interaction has to be the neutral gas coma population, not the solar wind minor ion flux. In other comets, the neutral gas coma has been collisionally thick, leading to the classical "crescent shaped" emission region between the nucleus and the Sun.

Further evidence for a relatively low rate of charge exchange interaction can be found in the overall scale of the observed emission region. The Chandra signal emission can be traced out

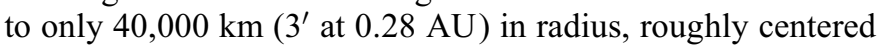
around the Sun-nucleus line. Similar to the 1997 measurements, the emission region is over an order of magnitude more compact than found in other comets; $80 \%$ of the emission was found within a spherical region of radius $40,000 \mathrm{~km}$ from the nucleus. Previous comets have demonstrated emission out to $10^{6} \mathrm{~km}$, the approximate limit of the neutral gas coma.

The sunward morphology in 2003 is different from that exhibited in 1997 July (Fig. 1d), with the 1997 structure appearing to be somewhat more crescent shaped. However, the 1997 optical coma also appears to be denser and more uniformly distributed. Differences in the effective spatial resolution cannot be the cause, as all three observations demonstrated "seeing" on the order of $10^{\prime \prime}$, limited by instrumental capabilities and photon counting statistics (Table 1).

\footnotetext{
${ }^{11}$ Data obtained from http://www.usc.edu/dept/space_science/semdata97.htm and http://umtof.umd.edu/pm/crn.

${ }_{12}$ See http://sd-www.jhuapl.edu/ACE/ULEIS/spec_gadget.html.
} 


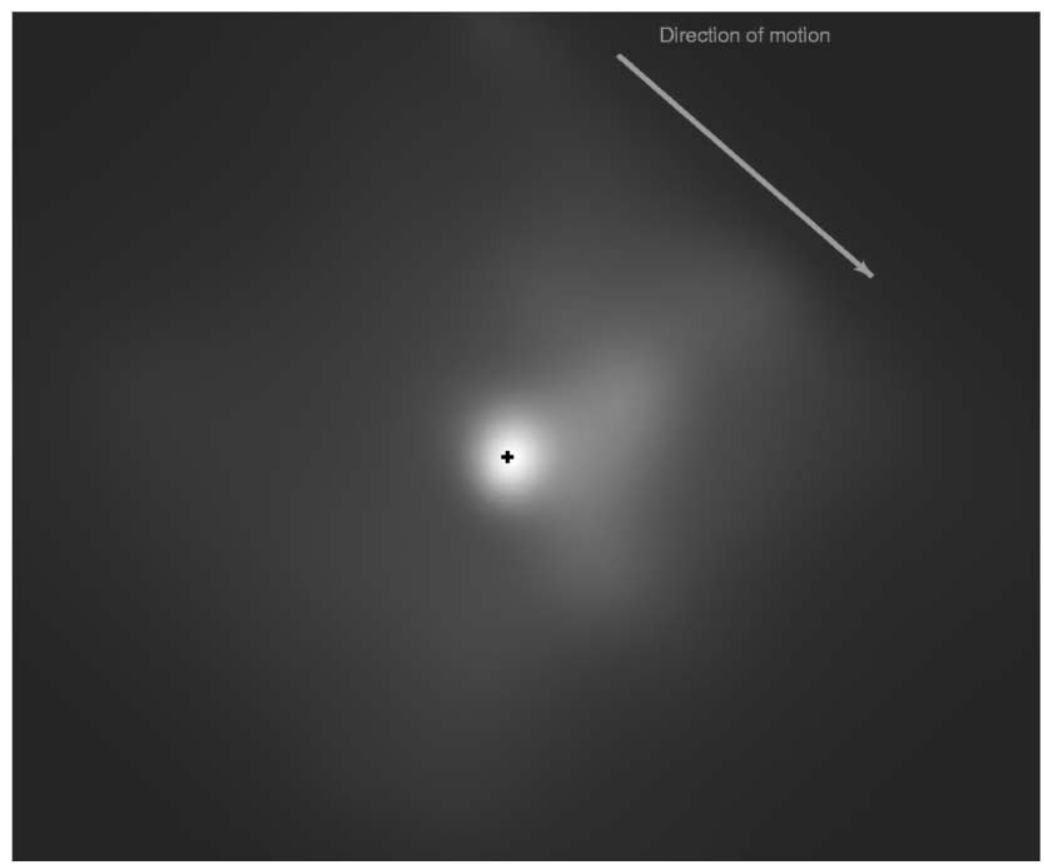

Fig. $1 a$

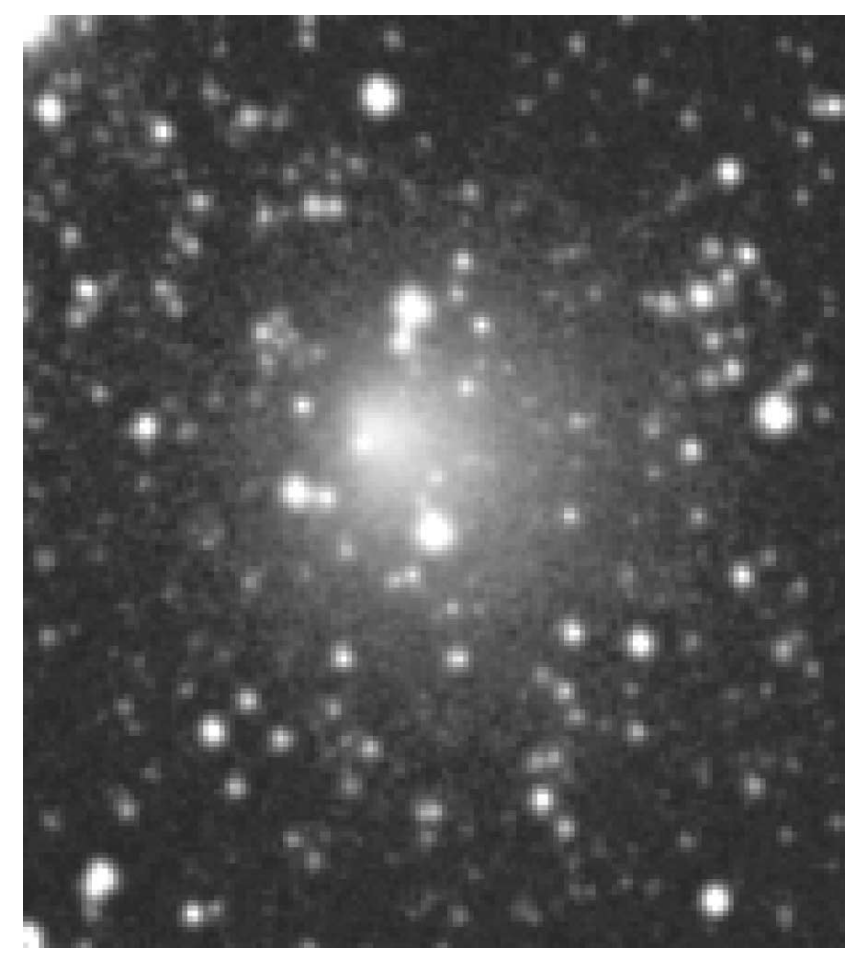

Fig. $1 b$

FIG. 1.-Morphology of the cometary X-ray emission. (a) Combined Chandra X-ray map of Encke on 2003 November 24. The photons have been reregistered from the Chandra detector coordinate system to a cometocentric coordinate system. North is up and east is to the left. The direction to the Sun is approximately toward the right. The arrow denotes the comet's direction of apparent motion. The cross denotes the position of the nucleus. The reconstructed field of view is $10^{\prime} \times 10^{\prime}\left[\left(9 \times 10^{4} \mathrm{~km}\right) \times\left(9 \times 10^{4} \mathrm{~km}\right)\right]$, and the pixel resolution is $10^{\prime \prime}$. (b) Wide-field optical green filter CCD image of the dust coma obtained by M. Jager and G. Rhemann on 2003 November 17 UT. (c) CN image of the gas coma on 2003 November 24 from D. Schleicher and L. Woodney. In both images, the comet exhibits a diffuse fan in the sunward direction and a centralized core of optical emission. Unlike other comets observed to date and Encke as observed in 1997 (Lisse et al. 1999), the X-ray and optical images correlate well. There is no obvious anti-sunward optical emission, nor is a tail evident. Encke appears to have been very inactive at the time of Chandra observation. (d) X-ray, EUVE, and optical images of Encke in 1997, after Lisse et al. (1999). Left: ROSAT HRI 1997 July 4-8 image. Middle: EUVE Lexan B 1997 July 6-8 image. The images are in a reference frame moving with the comet's apparent sky position. The projected direction toward the Sun is to the right in each image. Right: Visible-light image of the comet taken on 1997 June 4 UT, demonstrating a coma with asymmetric emission and a weak tail. The coordinate system and length scale of $1300 \mathrm{~km} / 10^{\prime \prime}$ pixel are the same in all three images. (e) Spatial profiles in the north-south direction, perpendicular to the nucleus-Sun line, for the X-ray emission (diamonds) and for the $\mathrm{CN} / \mathrm{OH}$ gas coma fluorescence (solid line) and dust coma continuum scattered radiation (dashed line) from CCD imagery obtained at the Lowell 42 inch $(1 \mathrm{~m})$ telescope by D. Schleicher and L. Woodney in 2003 . The X-ray emission profile, while noisy, tracks the $\sim 1 / \rho$ gas emission out to large distances, while the dust emission decreases much more rapidly in the core region. Here $3^{\prime}=40,000 \mathrm{~km}$, and the maximum offset between the peaks of the gas coma flux and the X-ray flux is within $2^{\prime \prime}$, or $450 \mathrm{~km}$. [See the electronic edition of the Journal for a color version of (a).] 


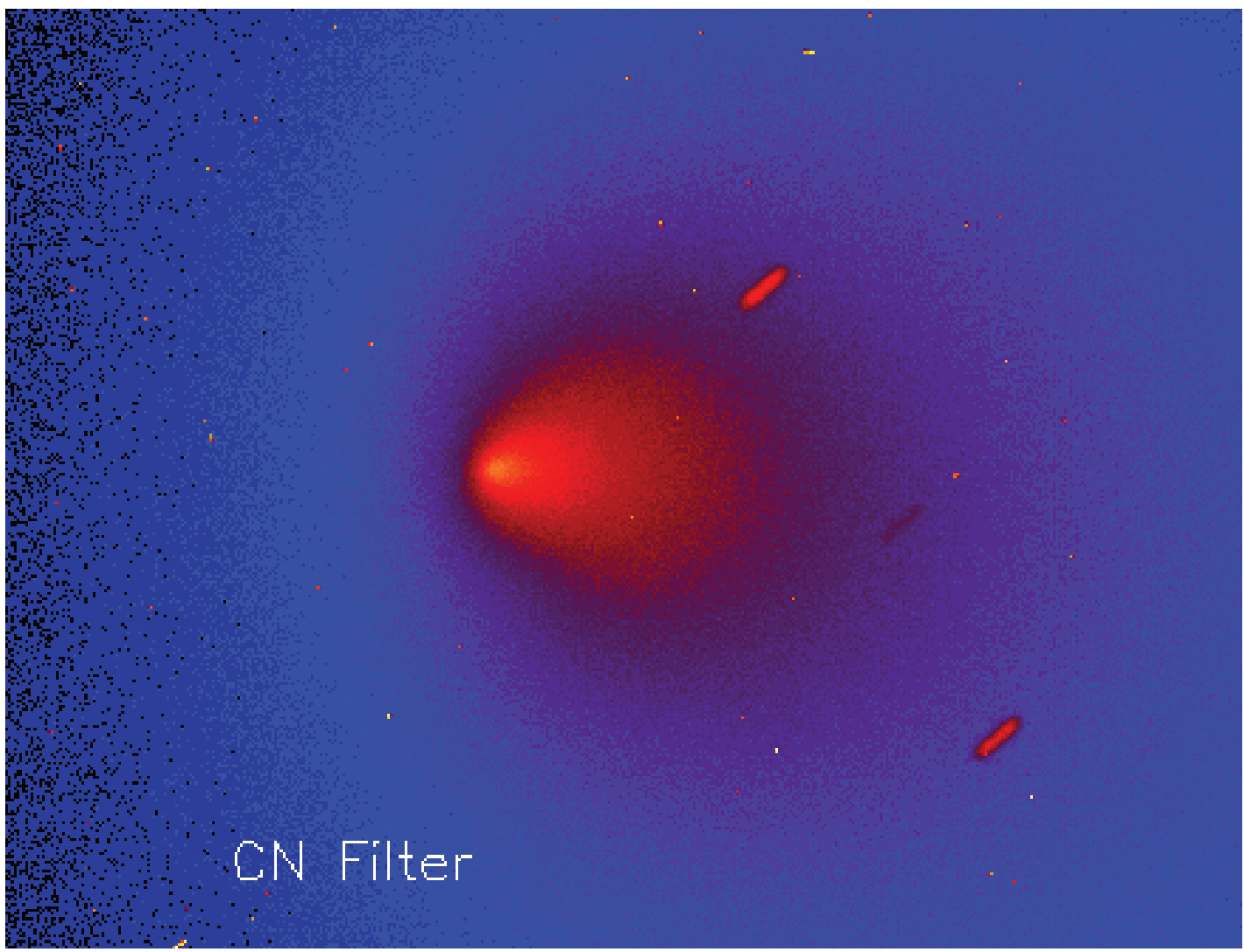

FIG. $1 c$
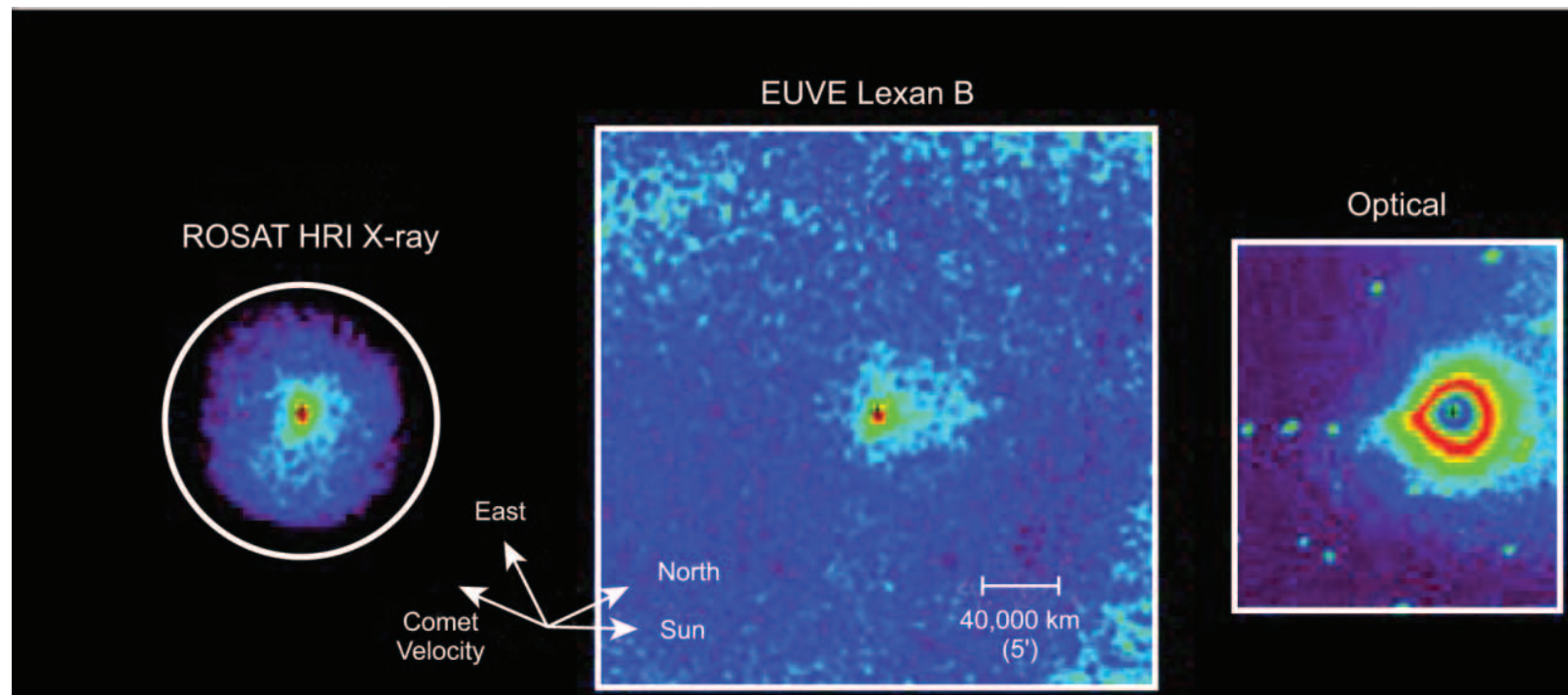

c)

With the comet at $0.27 \mathrm{AU}$ from the Earth, $1^{\prime \prime}$ corresponded to $215 \mathrm{~km}$ at the nucleus, so the theoretical Chandra resolution at the comet was $100 \mathrm{~km}_{\text {pixel }}{ }^{-1}$. However, the actual size of a statistically significant spatial resolution element was determined by the limited number of photons detected. In regions of high net signal, e.g., near the nucleus, this resolution was on the order of $2^{\prime \prime}(430 \mathrm{~km})$ per resolution element. The average photon- limited effective pixel size realized over the detected X-ray emission region was $7^{\prime \prime}(1500 \mathrm{~km})$ per resolution element.

Light curve.-The source had a total of 6500 counts in the 300-1000 eV energy range after background removal, corresponding to a total $0.3-1.0 \mathrm{keV}$ X-ray luminosity $L_{\mathrm{X}}$ of $3.8 \times$ $10^{14} \mathrm{ergs} \mathrm{s}^{-1}$ and $E_{\text {photon }}=450 \mathrm{eV}$ (and an average Chandra effective area of $50 \mathrm{~cm}^{2}$ ). This is similar to the $4 \times 10^{14} \mathrm{ergs} \mathrm{s}^{-1}$ 


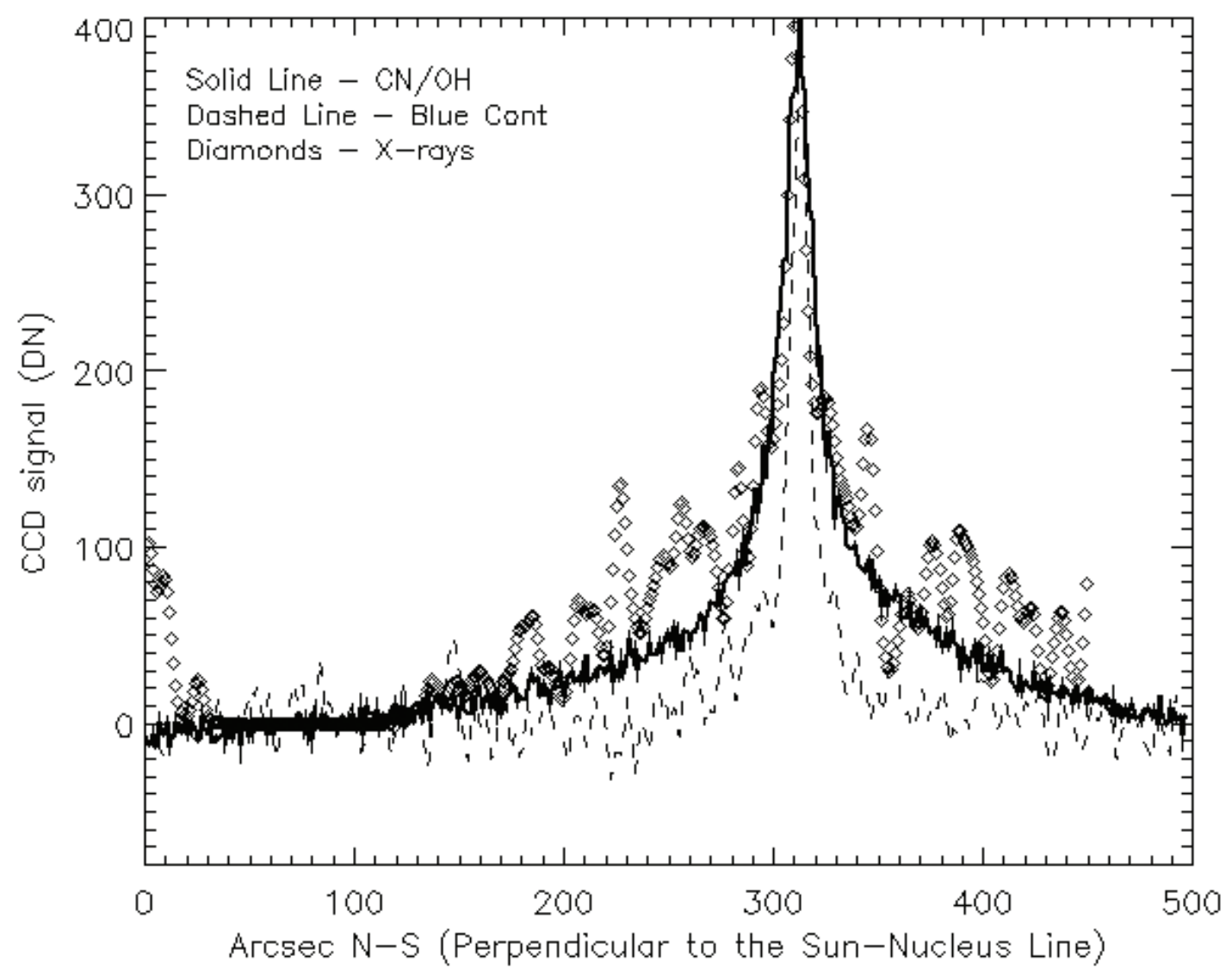

FIG. $1 e$

found for Encke in 1997 using the ROSAT HRI from 0.10 to $0.50 \mathrm{keV}$, with an average photon energy of $0.23 \mathrm{keV}$. (N.B., the ROSAT observations, being insensitive to the bright cometary emission lines from 0.5 to $0.8 \mathrm{keV}$, probably underestimated the Chandra luminosity by about a factor of 3; see Fig. 3.) The realized Chandra count rate is roughly 1 order of magnitude lower than expected, due to a combination of the degraded Chandra soft X-ray system response in Cycle 5 from focal plane surface contamination, the low solar wind flux density at the time of observation, and the relatively low level of cometary outgassing activity at the time of observation. The average total count rate, after background removal, was $\sim 0.12$ counts $\mathrm{s}^{-1}$.

A light curve for the Chandra observations on 2003 November 24 was created by binning all the $300-1000 \mathrm{eV}$ events in a $40,000 \mathrm{~km}$ radius aperture into 30 discrete intervals, corresponding to the 30 pointings of the spacecraft performed during the
$15 \mathrm{hr}$ of on-target time (Fig. 2a). The linearly decreasing trend seen in the solar wind flux (solar wind flux $=$ density $\times$ velocity; Fig. 4c) was then removed. The resulting light curve demonstrates a periodicity at $10.5 \pm 1.5 \mathrm{hr}(2 \sigma)$ and a peak-to-peak amplitude of $20 \%$. The majority of the error found in our light curve measurements was due to fluctuations of the background and in fitting the solar wind flux trend with time. We are able to rule out the null variation case (periodicity $=0 \mathrm{hr}$ ) at the $95 \%$ confidence limit. The variation of the background signal removed from the observations does not show any periodicity. None of the impulsive events found in our previous observations of comets was seen (Lisse et al. 1996, 1999, 2001).

Spectroscopy. - The Chandra ACIS-S spectra (extracted from a circular aperture 3.7 or $43,000 \mathrm{~km}$ in radius, centered on the nucleus position) show clear evidence of line emission at the appropriate energies for hydrogen-like and helium-like $\mathrm{C}, \mathrm{N}, \mathrm{O}$,

TABLE 1

Observation Times and Observing Parameters

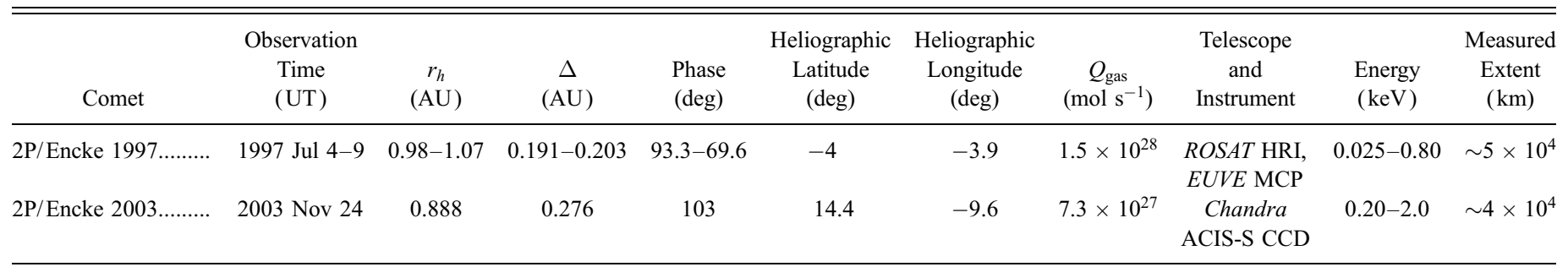

Notes.-Position, gas production rate, and approximate radial extent of the observed X-ray emission for Encke in 1997 and 2003 . Here $r_{h}$ is the comet-Sun dis-

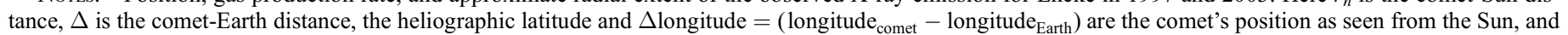
$Q_{\text {gas }}$ is the gas production rate from the nucleus surface (Wegmann et al. 2004; T. Mäkinen 2004, private communication). 


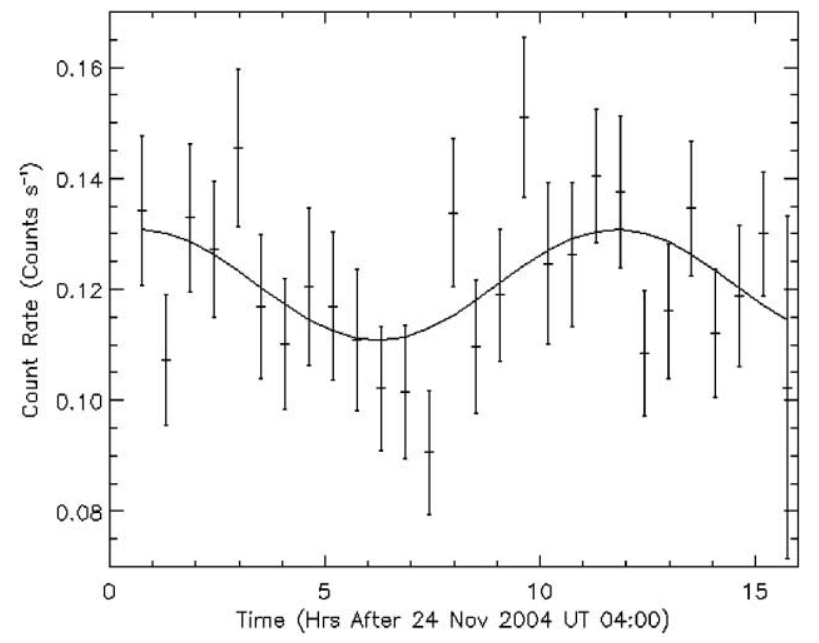

FIG. $2 a$

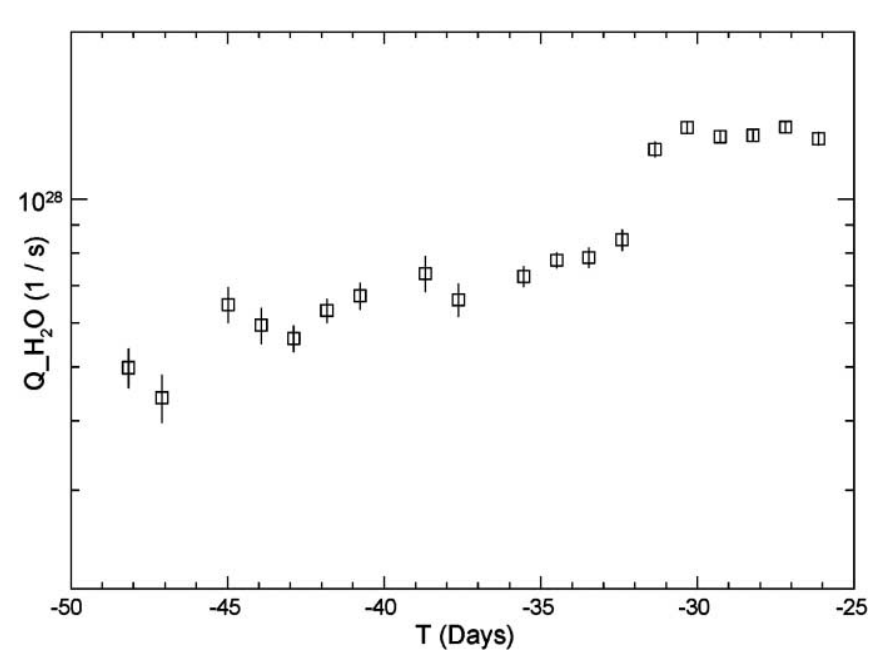

FIG. $2 b$

FIG. 2.-Time dependence of the X-ray emission. (a) Total $0.3-1.0 \mathrm{keV}$ count rate vs. time, grouped by the 30 pointings of the spacecraft on the comet. Dashes:

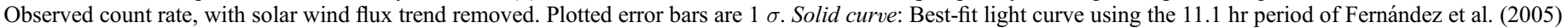

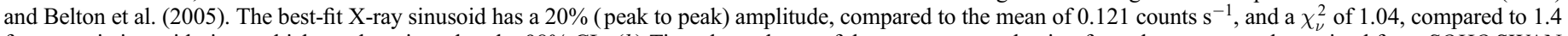

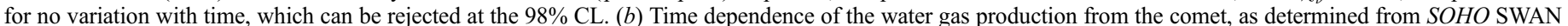

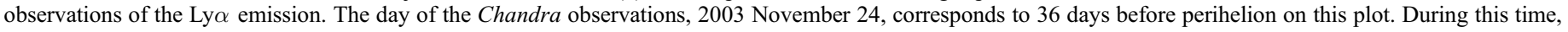
the water production was monotonically increasing as the comet approached the Sun.

and Ne solar wind ions (Fig. 3, Table 2). The strongest and most easily resolved emission in the Encke spectrum, as in other cometary spectra (Lisse et al. 2001; Krasnopolsky et al. 2002), are due to the $\mathrm{O}$ VII charge exchange complex at $550-580 \mathrm{eV}$, the $\mathrm{O}$ VII $/ \mathrm{O}$ VIII charge exchange lines at $\sim 660 \mathrm{eV}$, the $\mathrm{O}$ VIII lines at 780 and $830 \mathrm{eV}$, the $\mathrm{C}$ VI and $\mathrm{C} v$ charge exchange lines at 368 and $308 \mathrm{eV}$, the NV VIII/C VI line complex at $430-480 \mathrm{eV}$, and the $940 \mathrm{eV}$ Ne Ix charge exchange line. Comparison of the measured line centers in Table 2 and the theoretical line energies shows agreement between the predicted and measured line energies within the measurement accuracy, $\sim 0.05 \mathrm{keV}$, of the ACIS-S. The Chandra spectrum is consistent with the broadband X-ray photometry obtained in 1997 for comet Encke using ROSAT and EUVE (Lisse et al. 1999).

While the best model fit to the data was found for a model with eight emission lines due to $\mathrm{C}, \mathrm{N}, \mathrm{O}$, and $\mathrm{Ne}$, similar models with slightly different mixes of emission lines + continuum were also allowable fits to the data, at the 95\% CL (Table 2). Despite the Chandra results on comets C/1999 S4 (LINEAR) (Lisse et al. 2001) and C/McNaught-Hartley 2001 (Krasnopolsky et al. 2002; Kharchenko et al. 2003), it is still not clear how many charge exchange lines are readily detectable in the 250-1000 eV energy range. While the $\mathrm{C} / 1999 \mathrm{~S} 4$ (LINEAR) and $\mathrm{C} / \mathrm{McNaught}-\mathrm{Hartley}$ 2001 results clearly show line emission due to charge exchange, they were limited by photon statistics and unable to distinguish, at the $95 \% \mathrm{CL}$, between four- and six-line + continuum models and a MEKAL model with variable CNO abundance. Similarly, the 6500 total counts found for Encke do not allow any more elucidation of the nature of the background. At eight lines, we suspect that we have detected the maximum number of lines statistically possible in our 200-1000 eV moderate-resolution spectrum. The observed "continuum" should be understood to be the blending of many weaker lines, with possibly some small amount of true continuum emission due to thermal bremsstrahlung continuum or solar X-ray scattering.

The most striking result of the spectroscopic measurements is the strength of the $\mathrm{C}$ IV and $\mathrm{C} v$ lines in the $300-500 \mathrm{eV}$ region. They are as strong as the line blend of $\mathrm{O}$ VII triplet $+\mathrm{O}$ VIII found at $\sim 560 \mathrm{eV}$. For comets C/1999 S4 (LINEAR) and C/McNaughtHartley 2001, the oxygen lines were $\sim 3$ times stronger than the carbon lines. From the spectrum alone, it is clear that Encke represents a very different spectral regime for cometary X-ray emission.

Other observations of $2 P /$ Encke 2003. - An extensive series of observations of Encke were taken by the cometary community to take advantage of the exceptional 2003 apparition; these observations allow us to compare the observed X-ray emission with other properties of the comet.

SOHO SWAN.-The SOHO SWAN Ly $\alpha$ imager is a powerful tool to study the water gas production rate of comets (Mäkinen et al. 2001). Unfortunately, despite requests to the $S O H O$ project, there were no pointed observations of Encke taken within several weeks of the Chandra observations. Instead, we were left with only SWAN full-sky images to analyze. This was not, however, a significant shortcoming since the comet was fairly bright at the time versus the background. For the first half of November up to and including November 22, the comet remained in an area of sky with very heavy UV star contamination, but fortunately it moved to a lower background region just in time for the Chandra event. Interestingly, on November 28 there appears to be a moderate outburst that dies off by the end of the analyzed period. Taking these issues into account, we find a water production rate of $5.2 \times$ $10^{27} r_{h}^{-2.75} \mathrm{~s}^{-1}$ for the whole $\mathrm{SOHO}$ observation period in late November of 2003 , with $(7.3 \pm 0.3) \times 10^{27} \mathrm{~s}^{-1}$ for the day of the Chandra observation (Fig. $2 b$ ). While the comet's activity was low compared to a "bright" comet, it was still significantly higher than the lowest ever measured for a cometary body, circa $10^{25} \mathrm{~s}^{-1}$. The water production for the comet was increasing daily through the day of the X-ray measurements, as the comet approached the Sun. No major outbursts in gas production were seen until 4 days after the Chandra measurements.

Spitzer Space Telescope.-W. T. Reach et al. (2004, private communication) obtained mid-infrared images of Encke before and after perihelion in 2003. While both images show a nucleus and extended dust trail, both permanent features of the comet, only the post-perihelion image shows evidence of a dust coma. 


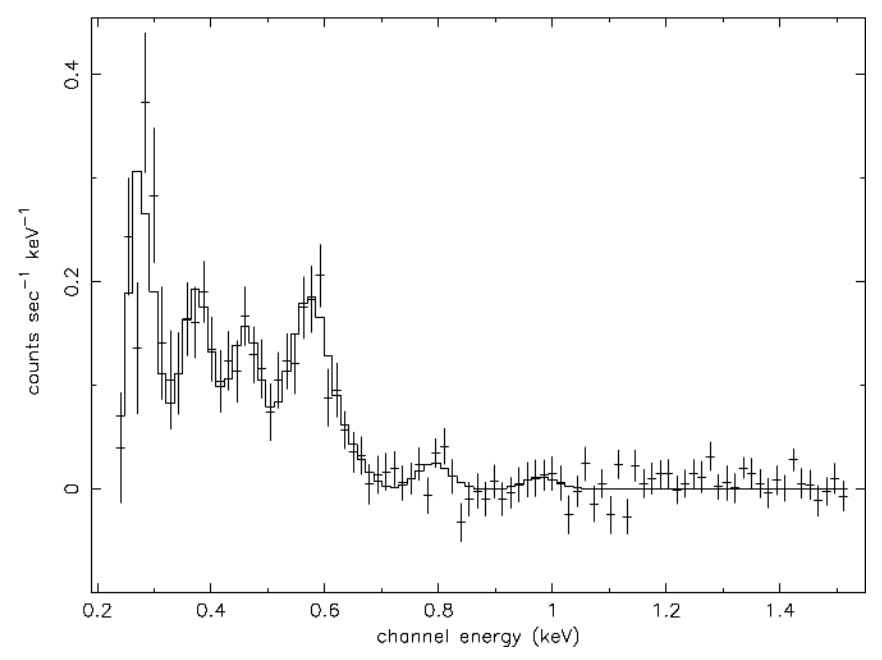

FIG. $3 a$

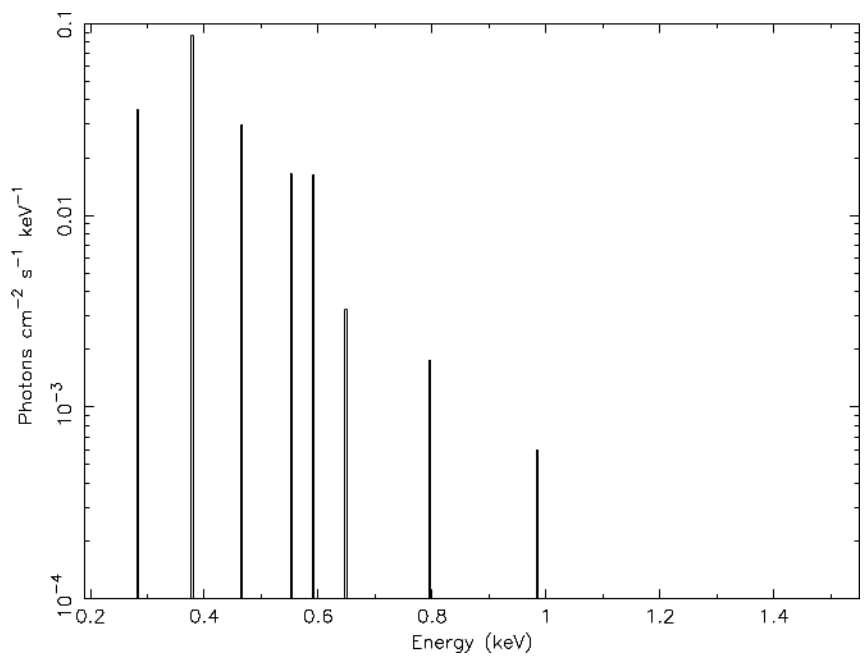

FIG. $3 b$

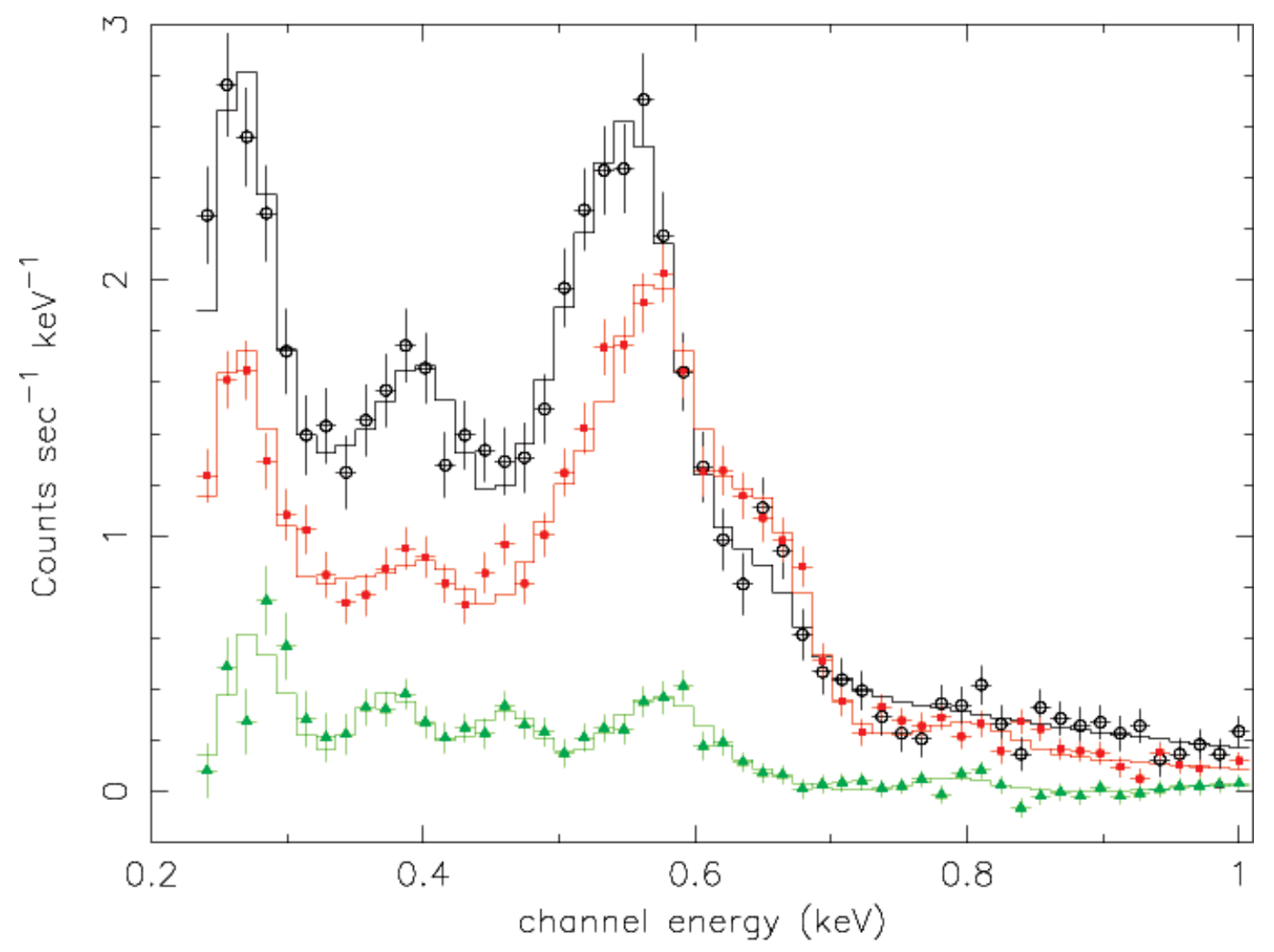

FIG. $3 c$

FIg. 3.-Spectra of the X-ray emission. (a) The $0.2-1.5 \mathrm{keV}$ pulse-height spectrum of the 2003 November 24 X-ray emission, as measured by the ACIS-S CCD S3 chip in direct-detection mode. Solid line: Best eight-line model fit to the emission (see Table 2 for parameter values). As for previous comets, pronounced emission due to $\mathrm{O}$ VIII and $\mathrm{O}$ VII is evident at 560 and $660 \mathrm{eV}$. Unlike other comets, the $\mathrm{C} \mathrm{VI}, \mathrm{C} \mathrm{v}$, and $\mathrm{N}$ vI emission lines in the $200-500 \mathrm{eV}$ range are as strong as the $500-700 \mathrm{eV} \mathrm{O}$ vII / $\mathrm{O}$ vIII lines. (b) Best-fit XSPEC eight-line emission model to the Chandra spectrum. The model lines at 284, 380, 466, 552, 590, 648, 796, and 985 are close to those predicted for charge exchange between solar wind $\mathrm{C}^{+5}, \mathrm{C}^{+6} / \mathrm{N}^{+6}, \mathrm{O}^{+7}, \mathrm{O}^{+8}, \mathrm{O}^{+8}, \mathrm{O}^{+8}$, and $\mathrm{Ne}^{+9}$ ions and neutral gases in the comet's coma. $(c) \mathrm{ACIS}$ cometary spectra of three comets. All curves show ACIS-S3 measurements with $\pm 1 \sigma$ error bars and the best-fit eight emission line + thermal bremsstrahlung model convolved with the ACIS-S instrument response as a histogram. Red circles: ACIS spectra of C/1999 S4 (LINEAR), from Lisse et al. (2001). Black squares: Comet McNaught-Hartley spectra, after Krasnopolsky et al. (2002). Green triangles: Comet 2P/Encke 2003 spectrum from this work, multiplied by a factor of 2 . The C/1999 S4 (LINEAR) and C/McNaught-Hartley 2001 observations had an average count rate on the order 20 times as large, even though Encke was closer to Chandra and the Earth when the observations were being made. Note the $560 \mathrm{eV}$ complex to $400 \mathrm{eV}$ complex ratio of $2: 3$ in the bright comets. 
TABLE 2

Spectral Model Fits

\begin{tabular}{|c|c|c|c|c|c|c|c|c|c|c|c|c|}
\hline \multirow[b]{2}{*}{ Model } & \multirow{2}{*}{$\begin{array}{c}k T_{\mathrm{TB}} \\
(\mathrm{keV})\end{array}$} & \multicolumn{8}{|c|}{$E_{\text {line }}(\mathrm{eV})$} & \multirow[b]{2}{*}{$\chi^{2 / \text { dof }}$} & \multirow[b]{2}{*}{ dof } & \multirow[b]{2}{*}{ Comments } \\
\hline & & 1 & 2 & 3 & 4 & 5 & 6 & 7 & 8 & & & \\
\hline Power law .............................. & $3.3 \pm 0.1$ & $\ldots$ & $\ldots$ & $\ldots$ & $\ldots$ & $\ldots$ & $\ldots$ & $\ldots$ & $\ldots$ & 5.3 & 57 & Index free \\
\hline ТВ & $0.22 \pm 0.13$ & $\ldots$ & $\ldots$ & $\ldots$ & $\ldots$ & $\ldots$ & $\ldots$ & $\ldots$ & $\ldots$ & 4.1 & 57 & \\
\hline 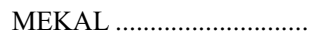 & $0.12 \pm 0.04$ & $\ldots$ & $\ldots$ & $\ldots$ & $\ldots$ & $\ldots$ & $\ldots$ & $\ldots$ & $\ldots$ & 3.4 & 57 & Solar abundances \\
\hline $\mathrm{TB}+$ four narrow lines..... & $0.26 \pm 0.07$ & 284 & 380 & 469 & 570 & $\ldots$ & $\ldots$ & $\ldots$ & $\ldots$ & 1.2 & 49 & Line centers free, line widths $=$ instrument resolution \\
\hline $\mathrm{TB}+$ four wide lines ......... & $0.27 \pm 0.2$ & 285 & 377 & 463 & 570 & $\ldots$ & $\ldots$ & $\ldots$ & $\ldots$ & 1.02 & 45 & Line centers and widths free \\
\hline $\mathrm{TB}+\operatorname{six}$ lines ...................... & $0.14 \pm 0.1$ & 286 & 375 & 463 & 532 & 581 & 636 & $\ldots$ & $\ldots$ & 1.08 & 45 & Line centers free, line widths $=$ instrument resolution \\
\hline 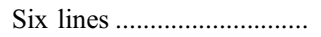 & $\ldots$ & 283 & 379 & 464 & 553 & 600 & 795 & $\ldots$ & $\ldots$ & 0.99 & 47 & No continuum \\
\hline Eight lines ........................... & $\ldots$ & 284 & 380 & 466 & 552 & 590 & 648 & 796 & 985 & 0.96 & 43 & No continuum \\
\hline
\end{tabular}

Notes.-Model spectral fits to the Chandra ACIS-S observations of $2 \mathrm{P} /$ Encke on 2003 November 24 . Here $k T_{\mathrm{TB}}$ is the effective energy for the thermal bremsstrahlung continua in all models except for the power-law models, where it is the index of the power law. The quantity "dof" is the number of degrees of freedom of the fit, equivalent to the number of independent spectral channels in the Chandra observations minus the fit parameters. The effective hydrogen column density between Chandra and the comet, $N_{\mathrm{H}}$, has been set to $10^{13} \mathrm{~cm}^{-2}$ for the fits, a value consistent with the estimated column between the Earth and the comet and essentially indistinguishable from zero for spectral fits of X-ray data.

As a proxy for the overall activity of the comet, the dust coma shows that Encke was much more active post-perihelion in 2003. Encke is known to have large "post-/pre-perihelion activity asymmetry" of approximately a factor of 2 (Sekanina 1988a, 1988b), most likely due to "seasonal effects" or insolation of actively emitting regions during only part of its orbit. Assuming the 2003 apparition to be representative, these active regions become more active after perihelion. The most likely explanation for this finding is that the broad sunward fan-jet, seen weakly in the pre-perihelion 2003 optical images, is much more active postperihelion and "fills in" the sunward coma, while pre-perihelion the coma is mainly located in the two directed jets (see Festou \& Barale 2000). Some of the difference in morphology and luminosity between the 1997 ROSAT/EUVE observations of the comet (taken 7 weeks after perihelion) and the 2003 Chandra observations of the comet (taken 6 weeks before perihelion) (Fig. 1) can then be understood as a difference in coma gas densities: there was much more neutral material, and more extension of the material in a uniform coma, in the postperihelion observations of 1997 July than the pre-perihelion observations of 2003 November.

Lowell Observatory. - As Encke was expected to be a bright comet in late 2003, D. Schleicher of Lowell Observatory included long-term narrowband photometry monitoring of Encke in his ongoing survey of comets. For our analysis, we received from their group images of the gas and dust comae of the comet in the $\mathrm{OH}, \mathrm{CN}$, and "blue continuum" filters on November 24 (Figs. $1 b$ and $1 c$ ). In all images, the comet exhibits a diffuse fan in the sunward direction and a centralized core of optical emission. The $\mathrm{CN}$ and $\mathrm{OH}$ images are almost identical in structure, except for the much lower signal-to-noise ratio of the $\mathrm{OH}$ image, which is intrinsically less bright and which suffers from terrestrial atmospheric absorption. The dust coma is centered more on the nucleus and has less sunward extension than the gas coma and the X-ray emission (Fig. 1e). Comparing the 2003 and 1997 optical continuum images, the 2003 image shows a weaker coma with directed emission centered mainly in two jets approximately $90^{\circ}$ apart, without any obvious tailward extension, while the 1997 image shows a well formed, rounded coma with a long, extended tail. Both images show a sunward emission asymmetry.

Solar wind characteristics at the time of the Chandra observations. - The solar wind environment at the time of observations was originally quite uncertain. Twenty-eight days before the time of observation, one of the largest X-class flares ever observed erupted from the corona, causing massive auroae on Earth and downtime for a number of spacecraft, including Chandra. There was much concern that 1 solar rotation (or 28 days) later, the active region responsible would rotate back toward the Earth during our observations. However, by the time of our observations, the Sun had entered a relatively quiescent phase; no solar flares are obvious in the solar X-ray flux during our observations, and SOHO LASCO images of the Sun are typical for a quiet Sun. There were, however, a series of small perturbations in the solar wind created by a number of coronal holes in the week before. The last perturbation of the solar wind occurred more than 3 days before our observations. Figures $4 a$ and $4 b$ show a simulation in the ecliptic plane of the interplanetary magnetic field polarity and solar wind velocity, density, and dynamic pressure at 00:00 and 12:00 UT on 2003 November 24 based on the studies of Dryer (1998) and Dryer et al. $(2001,2004)$ and the numerical procedure of Fry et al. $(2001,2003,2004)$. Encke and the Earth are located in a region of low-density, fast-moving material left in the wake of the propagating shock.

The time delay observed between an element of the corotating solar wind arriving at the Earth (as measured by the nearEarth $A C E, S O H O$, and Wind spacecraft) and the observed X-ray emission from the comet can be predicted by assuming a latitudeindependent solar wind flow, a quadrupole solar magnetic field, and propagation of the sector boundaries radially at the speed of the solar wind and axially with a period of one-half the solar rotation period of 28 days:

$$
\begin{aligned}
\Delta t_{\text {total }}=\Delta t_{\text {Carr }}+\Delta t_{\text {rad }}= & \frac{\text { longitude }_{\text {comet }}-\text { longitude }_{\text {Earth }}}{14^{\circ} 7 \text { day }^{-1}} \\
& +\frac{r_{\text {comet }}-r_{\text {Earth }}}{v_{\mathrm{SW}} \times\left(86,400 \mathrm{~s} \mathrm{day}^{-1}\right)} .
\end{aligned}
$$

For our Chandra observations, we purposely chose a time when the comet was closest to the Sun in order to maximize the comet's X-ray luminosity, while keeping the phase angle of observation near $90^{\circ}$ and the Earth-comet distance near its minimum value. This implied a comet closer to the Sun than the Earth and, for Encke, a comet that was in the retrograde sense from the Earth, earlier in the Carrington spiral than the Earth. The sum effect of the radial and longitudinal placement of the comet in the latitude-independent model is for a parcel of solar 

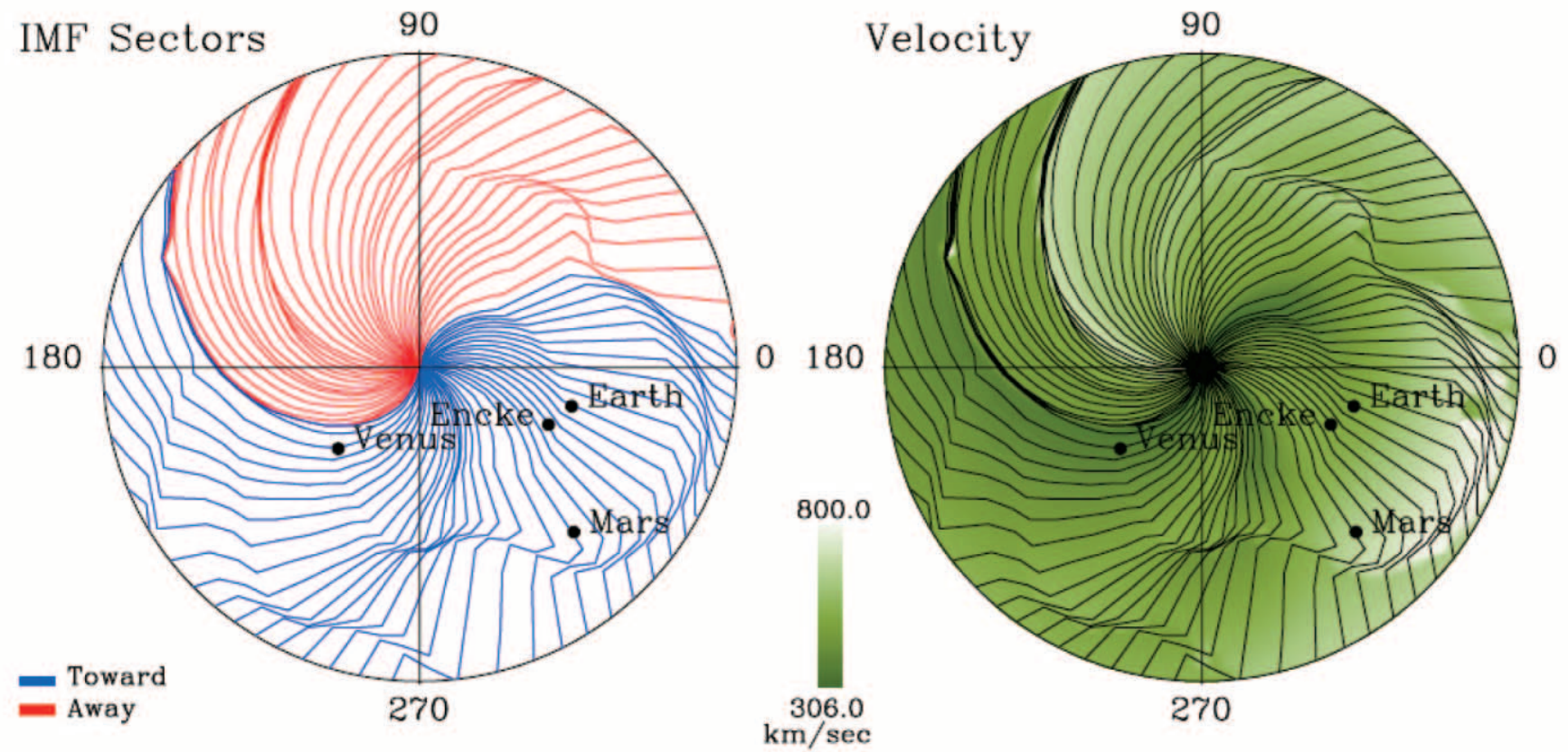

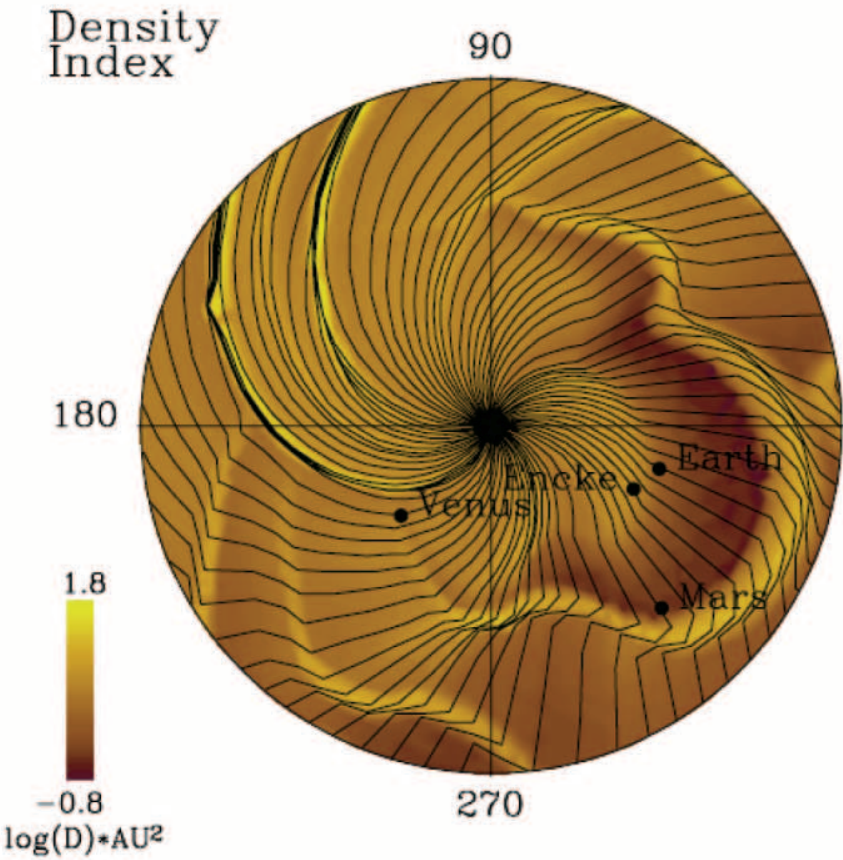

Nov 24, 2003

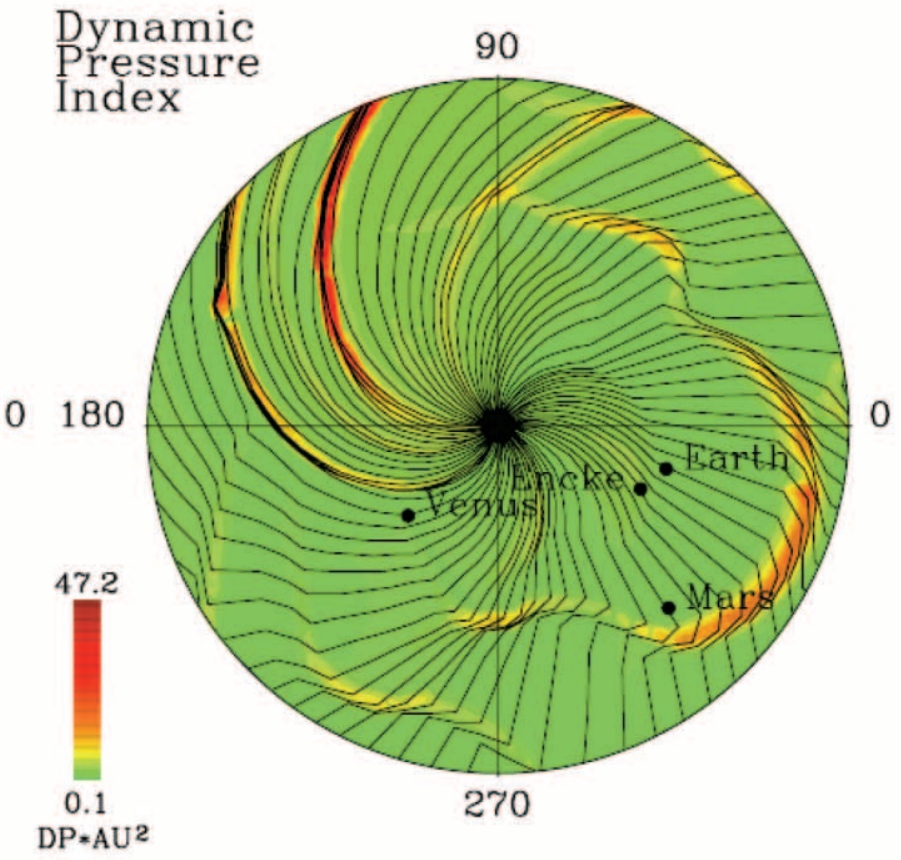

TIME $=00 \mathrm{UT}$

FIG. $4 a$

FIG. 4.-Structure of the interplanetary magnetic field, velocity profiles, and density and dynamic pressure contours. (a) Model contours on 2003 November 24 00:00 UT for the ecliptic plane between 0 and $2 \mathrm{AU}$ (Dryer et al. 2004). The view is from the north ecliptic pole. Encke's position 5 hr before the time of the Chandra observations, relative to Earth and several planets, is indicated by a small dot. For the duration of the Chandra observations, the comet resides in an atypical region of unusual but stable solar wind and magnetic field between two shocks propagating rapidly outward from the Sun. A third shock is beginning to form very close to the Sun. The normal pattern due to Carrington rotation can be seen in the upper left of each figure. (b) Same as in (a), except $12 \mathrm{hr}$ later at 2003 November 24 12:00 UT. Comparing the models for the two times, the propagation of the quiet region past Encke can be seen. (c) Solar wind parameters during the Encke observations. For the geometry of observation, the solar wind at the comet on November 24 encountered the Earth and $A C E / S O H O$ on November 25, approximately $26 \mathrm{hr}$ later ( Table 3 ). The panels show $A C E$ solar wind proton trends during the observations. The solar wind behavior was slowly changing, following a 3 day trend, with a moderate density slowly increasing from 4 to $5 \mathrm{~cm}^{-3}$, a high velocity slowly decreasing from 620 to $550 \mathrm{~km} \mathrm{~s}^{-1}$, and a thermal temperature decreasing from $\sim 1.5 \times 10^{6}$ to $1 \times 10^{6} \mathrm{~K}$. Assuming that the longitudinal + radial delay model given in the text is correct, the Chandra observations should correspond to ACE measurements from 02:40 to 17:40 UT on 2003 November 25 (Table 4). 

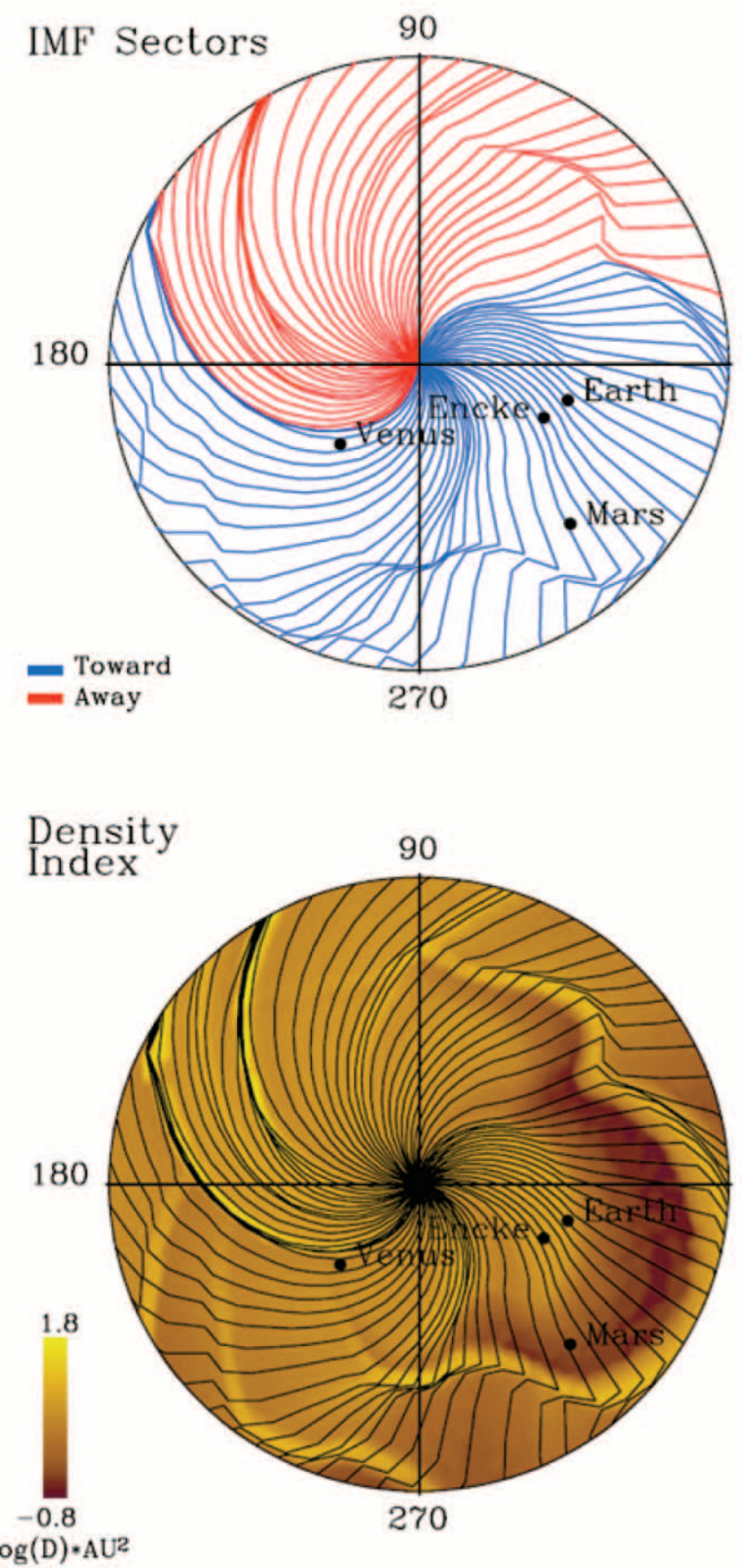

Nov 24, 2003
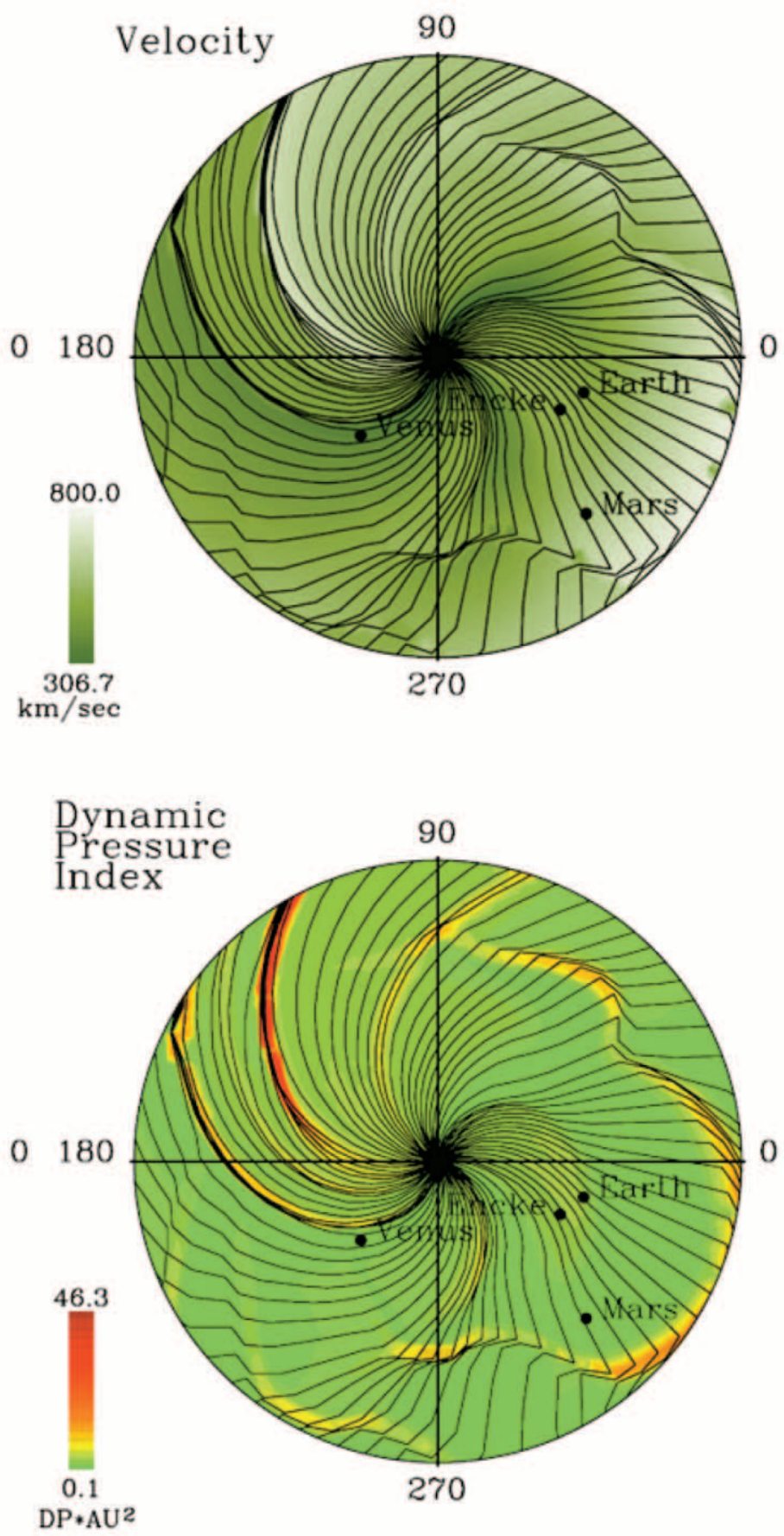

TIME $=12$ UT

FIG. $4 b$

wind to impact Encke 0.95 days $(22.8 \mathrm{hr})$ before arriving at the Earth (Table 3).

Using this timing, we find from the $A C E$ trend data that the solar wind density on 2003 November 24 at the comet was a low $3.5 \pm 1.5 \mathrm{~cm}^{-3}$, the solar wind speed was an elevated $600 \pm$ $40 \mathrm{~km} \mathrm{~s}^{-1}$, and the thermal temperature an abnormally high $(2 \pm 1) \times 10^{6} \mathrm{~K}$, more typical of the high solar corona than normal (Fig. 4c). $A C E$ charge state ratios for the solar wind are intermediate between the values expected for the slow and fast solar winds (Table 4). This is consistent with a solar wind containing a mix of hot and cold wind material, due to the coronal hole, from the base and top of the corona. The absence of impulsive events in the comet's X-ray light curve is consistent with high-speed solar wind flows. The solar wind parameters as determined by $A C E$ are also in good qualitative agreement with the solar wind state as deduced from the Encke X-ray spectrum. The high particle speed directly affects the $380 \mathrm{eV} \mathrm{C/560} \mathrm{eV}$ $\mathrm{O}$ line ratio in the Chandra spectrum, as does the unusual charge 


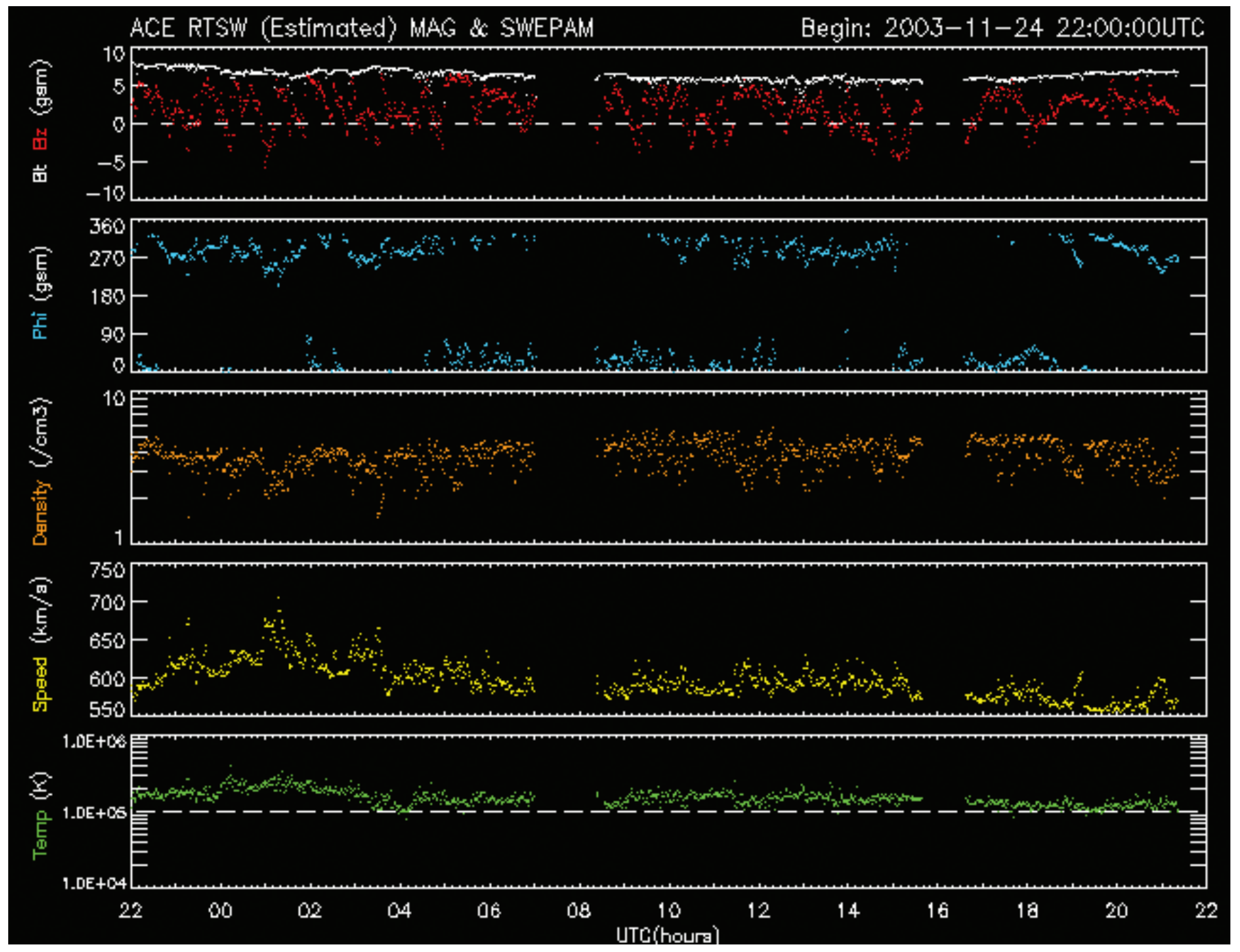

FIG. $4 c$

state, elevating the importance of the carbon lines (Fig. 3). The initial population of excited states $(n l)$ following charge exchange is strongly dependent on the collision velocity.

The dominant trend in the solar wind parameters was a gentle cooling and de-acceleration, as the solar wind relaxed from the coronal mass ejection back to the normal parameters for the equatorial wind. We were fortunate that the solar weather was benign and calm during the time of our observations; any major changes over time observed in the X-ray emission were due to something other than variations in the solar wind on a large scale. On the other hand, the weather was "hot" enough so that the Encke X-ray spectrum was unique compared to all other spectra measured to date!

\section{DISCUSSION}

Morphology.-Compared to our previous observation of comet Encke in 1997 using ROSAT, we have succeeded in mapping the $\mathrm{X}$-ray emission around the comet on scales of $500 \mathrm{~km}$ in the core,

TABLE 3

Solar Wind Arrival Time Difference, Comet and Earth

\begin{tabular}{|c|c|c|c|c|c|}
\hline Comet & $\begin{array}{c}\text { Observation Time } \\
\text { (UT) }\end{array}$ & $\begin{array}{l}\Delta t_{\text {long }} \\
\text { (days) }\end{array}$ & $\begin{array}{l}\Delta t_{\text {radial }} \\
\text { (days) }\end{array}$ & $\begin{array}{l}\Delta t_{\text {total }} \\
\text { (days) }\end{array}$ & $\begin{array}{c}\Delta t_{\text {observed }} \\
\text { (days) }\end{array}$ \\
\hline 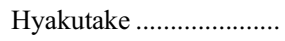 & 1996 Mar 27 & -0.23 & 0.032 & -0.20 & 0.24 \\
\hline Hale-Bopp ……................... & 1996 Sep 11 & -4.60 & 5.9 & 1.30 & +1.4 \\
\hline Tempel-Tuttle ..................... & 1998 Jan 29 & -2.31 & 0.37 & -1.94 & -2.5 \\
\hline 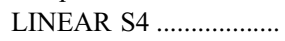 & $2000 \mathrm{Jul} 15$ & +1.2 & -0.30 to -0.50 & +0.9 to +0.7 & -0.25 \\
\hline 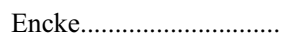 & 1997 Jul 7 & -0.26 & 0.093 & -0.17 & -0.1 \\
\hline 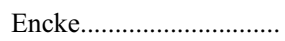 & 2003 Nov 24 & -0.65 & -0.30 & -0.95 & $\mathrm{~N} / \mathrm{A}$ \\
\hline
\end{tabular}

Noтеs.-Predicted and observed light curve phase shifts using the simple latitude-independent model. The estimated time shifts assume solar wind velocity as measured near Earth. Positive time shifts imply that the solar wind impulse happens at Earth first, comet next. Negative time shifts imply the solar wind boundary hits comet first, Earth next. 
TABLE 4

aCE Solar Wind Charge State Ratios during the Encke Observations

\begin{tabular}{clcc}
\hline \hline Species & $A C E$ & $\begin{array}{c}\text { Schwadron \& Cravens } \\
\text { Fast Wind }\end{array}$ & $\begin{array}{c}\text { Schwadron \& Cravens } \\
\text { Slow Wind }\end{array}$ \\
\hline $\mathrm{O}^{7+} / \mathrm{O}^{6+} \ldots \ldots \ldots \ldots . .$. & 0.1 & 0.03 & 0.27 \\
$\mathrm{C}^{5+} / \mathrm{C}^{6+} \ldots \ldots \ldots \ldots .$. & 2.38 & 5.18 & 0.66 \\
\hline
\end{tabular}

Notes.-Measured $A C E$ solar wind minor ion charge state ratios on 2003 November 25, as compared to the average ratios for the fast and slow solar winds listed in Schwadron \& Cravens (2000). Despite the fact that Encke is a low-latitude comet, where the slow solar wind is expected to dominate, the measured ratios are closer to the average of the fast and slow wind values.

high-signal region near the nucleus and on scales of $1500 \mathrm{~km}$ per resolution element throughout the coma. Imaging at these fine scales is unprecedented and is of great interest. Before these observations, it was not known whether the X-ray emission peaks at the position of the nucleus, or "in front" of the nucleus, toward the Sun, within an uncertainty of $\sim 1500 \mathrm{~km}$.

How the X-ray emission relates to the structures within the cometary atmosphere, such as the low Mach number bow shock and the region of plasma exclusion within the cometopause, is also poorly known, although recent work by Wegmann \& Dennerl (2005) in detecting the emission morphology discontinuity due to the bow shock region has begun to change this. Assuming an average charge exchange cross section for the coma neutral species of $\sim 3 \times 10^{-15} \mathrm{~cm}^{2}$ and hemispherical outflow for Encke, we find $\tau_{\text {charge exchange }} \sim 1$ for charge exchange at $1000 \mathrm{~km}$ from the nucleus. The minimum Chandra resolution element has a width of $\sim 2^{\prime \prime}$, or $430 \mathrm{~km}$. Thus the observed emission from the comet was collisionally thin to charge exchange except in the central two resolution elements containing the nucleus; this region accounted for less than $0.5 \%$ of the total observed emission from the comet. The position of the subsolar bow shock and contact surface for Encke during our observations can be inferred from the $\mathrm{P} /$ Halley in situ magnetic field measurements (Neugebauer et al. 1987), scaling the distances by the factor $Q_{\text {gas }}$ for each comet, where $Q_{\text {gas }}$ is the total production rate of gas from the nucleus (Flammer 1991; Lisse et al. 1999). Using a gas production rate for Halley of $1 \times 10^{30} \mathrm{~mol} \mathrm{~s}^{-1}$ and a bow shock distance of $5 \times 10^{5} \mathrm{~km}$ (Mukai et al. 1987; Reme et al. 1987), we find for Encke on 2003 November 24 a bow shock distance of $1000 \mathrm{~km}$ and the cometopause boundary to be at $\sim 120 \mathrm{~km}$ from the nucleus. While these locations are highly contracted compared to other comets due to the low rate of volatile emission and the corresponding low coma density, they should be readily discernible within the Chandra images with $430 \mathrm{~km}$ spatial resolution. We thus expected to be able to detect them positively in Encke's coma. However, we have not been able to find structures other than a strongly peaked maximum of the emission centered at the nucleus and a fan-shaped structure pointed toward the Sun, both of which mimic the gas coma structure more than anything else.

Or is this simple bow shock-cometopause model of the cometary plasma structure incorrect? In Figure 5 we show the results of a classical magnetohydrodynamic calculation (Gombosi et al. 1996; Häberli et al. 1997) of the interaction of the solar wind with a very asymmetric outgassing comet $2 \mathrm{P} /$ Encke. The model calculation assumes a gas production of $8 \times 10^{27} \mathrm{~s}^{-1}$, with $90 \%$ of the gas emitted into the sunward side, varying as the cosine of the subsolar angle, and 10\% emitted spherically. The ionization rate is $1.3 \times 10^{-6} \mathrm{~s}^{-1}$ at a heliocentric distance of 1 AU. The solar wind conditions assumed are a Parker spiral magnetic field with components $(-6,6,0) \mathrm{nT}$, a proton density of $4 \mathrm{~cm}^{-3}$, a velocity of $570 \mathrm{~km} \mathrm{~s}^{-1}$, and a temperature of $1.3 \times 10^{6} \mathrm{~K}$. The model-predicted bow shock distance is $25,000 \mathrm{~km}$ sunward of the comet and $\sim 50,000 \mathrm{~km}$ in the flanks, somewhat larger than we derived from linearly scaling from Halley's $Q_{\text {gas }}$ and bow shock position. The cometopause intersects the comet-Sun line at about $120 \mathrm{~km}$ sunward of the nucleus and is highly asymmetric. The dark blue interior region corresponds to the diamagnetic cavity, which excludes the outer magnetized solar wind and extends into the long thin ion tail. Comparing to the Chandra observations, we do not see an obvious correlation between the modeled plasma structures and the observed X-ray morphology, other than the circular emission region centered on the nucleus and extending out to the minima at $17,000 \mathrm{~km}\left(38^{\prime \prime}\right)$ radius (Fig. 1), close to the predicted position of the Encke bow shock. There is significant emission outside this radius, and there is no obvious tailward structure. Because the X-ray emission is produced by an interaction between the slowed high charge state solar wind ions and the neutral gas, it is expected that any spatial features associated with the plasma interaction would require an angular resolution smaller than the cometopause distance. Seeing plasma interaction effects for a weak comet such as Encke in an X-ray image may be further complicated by finite (large) gyroradius effects near the bow shock and finite (large) mean free path effects near the cometopause. Both effects tend to smear any strong discontinuities predicted by the pure MHD picture.

If instead we focus on the individual highly charged solar wind ions and their interaction with the neutral gas atmosphere of the comet, we find a very different answer. A detailed, threedimensional model of the nucleus+coma structure allowing for variable ionization rate of the outflowing coma gas with planar solar wind flow (Bodewits et al. 2004) projected onto the plane of the sky, and compared to the morphological structure found in the 1".5 resolution Encke X-ray images, is given in Figure $6 a$. In this model, the density distribution of water and its dissociation products is calculated using a standard Haser model with symmetrical hemispherical outflow and outstreaming gas velocity $v=0.85 r_{h}^{-1 / 2}$, where $r_{h}$ is the heliocentric distance. Evolution of the charge state distribution of the ions is followed as they capture electrons from cometary neutrals. In this model solar wind ions are allowed to penetrate right up to the nucleus; however, given the small size of the cometopause compared with the image resolution, this is not a serious problem. It is assumed that the ions follow straight-line trajectories and have an initial charge state distribution and composition taken from $A C E$. Using velocity-dependent total single electron capture cross sections from Fritsch \& Lin (1984) and Shipsey et al. (1983), charge state distributions for $\mathrm{C}^{5+}$ and $\mathrm{C}^{6+}, \mathrm{N}^{5+}$ to $\mathrm{N}^{7+}$, and $\mathrm{O}^{6+}$ to $\mathrm{O}^{8+}$ were calculated. As an example, the charge state distribution for oxygen and carbon ions along the comet-Sun axis is given in Figure $6 b$. It is immediately clear that Encke is collisionally thin to charge exchange, as there is still a significant amount of highly charged oxygen and carbon close to the nucleus.

The chief inputs to this model are the rate of emission of the neutral coma gas, $Q_{\text {gas }}$, and the charge state and velocity of the instreaming solar wind. For values of $Q_{\text {gas }} \geq 10^{29} \mathrm{~mol} \mathrm{~s}^{-1}$, we recover the "typical" crescent-shaped morphology with maximum offset toward the nucleus. In the collisionally thin regime, for low values of $Q_{\text {gas }}\left(<10^{28} \mathrm{~mol} \mathrm{~s}^{-1}\right)$ and nominal solar wind properties, the limiting factor is not the number of incoming solar wind minor ions, but the scarce neutral gas coma target molecules. The emission morphology does not show the characteristic crescent shape of the more active comets. Instead, the 


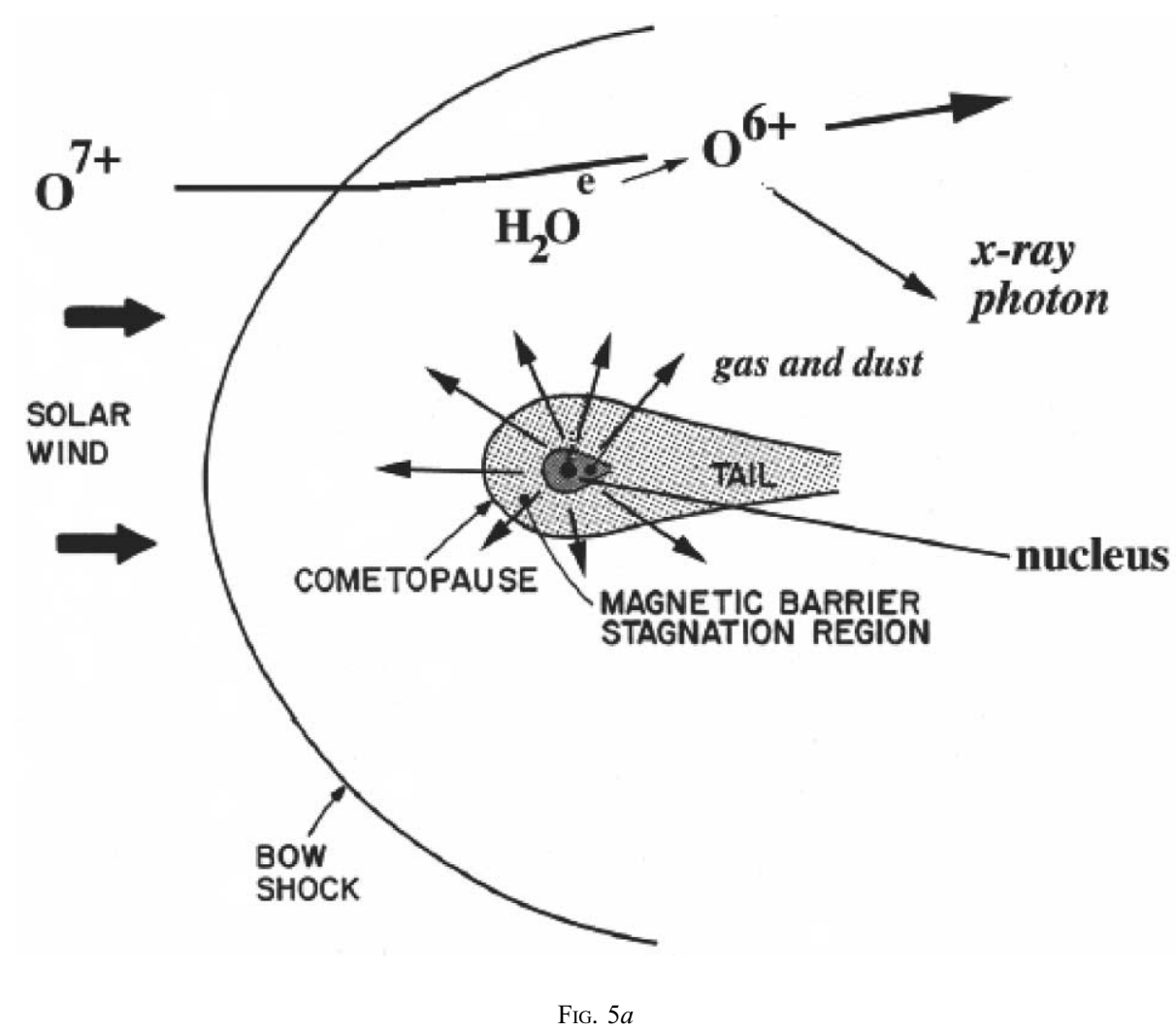

FIG. 5.-Predicted morphology of the plasma environment around comet 2P/Encke. (a) Schematic of the charge exchange interaction, following Cravens (2002). (b) Wide view of the plasma density distribution, with the position of the bow shock intersecting the comet-Sun line at about $25,000 \mathrm{~km}$ sunward of the nucleus, as predicted from an MHD calculation (Gombosi et al. 1996; Häberli et al. 1997). The values are plotted in the plane containing both components of the Parker spiral interplanetary magnetic field. The draping field lines are also shown. (c) MHD predictions of the magnetic field magnitude (color scale) and magnetic field line draping (lines with arrows) in the vicinity of the cometopause (shown as the yellow-white horseshoe-shaped line that separates the pure cometary ion flow inside from the mass-loaded solar wind flow outside).

model emissivity is a diffuse cloud with a maximum around the nucleus, where the density peaks and traces out the locations of the thin coma gas. Allowing for Encke's very nonsymmetric outgassing morphology, the prediction of a collisionally thin coma with charge exchange occurring wherever there are substantial quantities of neutral coma gas is consistent with the agreement we see between the 2003 images of the observed X-ray morphology and the optical coma (Fig. 1).

Comparing our 2003 Chandra observations to the 1997 EUVE/ROSAT observations of the comet, we find very different morphologies for the X-ray emission. The 1997 observations demonstrated the "classical" crescent-shaped emission pattern in the sunward half of the coma, produced by a collisionally thick interaction between the solar wind and the coma. Both times the comet was observed within $0.3 \mathrm{AU}$ of the Earth, and the estimated $\mathrm{SOHO}$ water production rates were the same within a factor of 2.5 ( T. Mäkinen 2004, private communication). The major difference seems to be that the 2003 observations were taken pre-perihelion, with cometary activity mainly found in the directed emission produced by two jets plus a weak fan (Figs. $1 b$ and $1 c$ ), while in 1997 the observations were taken after the comet had been thoroughly heated by a perihelion solar passage and a relatively dense, spherical coma had formed (Fig. 1d). Even allowing for the different observational capabilities of the ROSAT and Chandra X-ray cameras, these differences are real. Evidently, an important change in the observed morphology occurs at $Q_{\text {gas }} \sim$ $10^{28} \mathrm{~mol} \mathrm{~s}^{-1}$. We do find it surprising, however, that such large changes in behavior happen for only a factor of 2.5 difference in $Q_{\text {gas }}$.
Total X-ray luminosity.-As a check, we adopt the simple charge exchange model of Cravens (1997) to determine whether charge exchange is a plausible mechanism for the observed emission. From the model, we have $L_{\mathrm{X}} \sim 4.0 \times 10^{-21} N_{\text {neutral }} N_{\mathrm{SW}} \times$ $\left(v_{\mathrm{SW}} / 400 \mathrm{~km} \mathrm{~s}^{-1}\right) V \mathrm{ergs} \mathrm{s}^{-1}$. For Encke, using a hemispherical volume $V$ of radius $40,000 \mathrm{~km}$, a spherical gas outflow at $v_{\text {gas }}=$ $0.8 \mathrm{~km} \mathrm{~s}^{-1}$ and $Q_{\text {gas }}=7.3 \times 10^{27} \mathrm{~s}^{-1}$ (Table 1), and a $\mathrm{SOHO}$ solar wind density outside the bow shock of $\sim 4 \mathrm{~cm}^{-3}$, we find a total X-ray luminosity for the comet of $\sim 2.8 \times 10^{14} \mathrm{ergs} \mathrm{s}^{-1}$. This value is consistent with the measured $3.8 \times 10^{14}$ Chandra luminosity, within the errors of the Cravens model $( \pm 100 \%)$.

Temporal variations. - None of the impulsive events found in our previous observations of comets was seen (Lisse et al. 1996, 1999, 2001; Table 3). The solar wind flow in high-speed streams is known to be rather smooth, whereas the slow flow more typical near the ecliptic plane is known to be more turbulent (McComas et al. 1998). The $15 \mathrm{hr}$ duration of the observations may have been too short to expect a solar wind sector boundary crossing (period $\sim 7$ days) while we were conducting the Chandra observations. The 1997 ROSAT/EUVE observations demonstrated a 25\% duty cycle for the impulses over a 2 week time span.

In terms of our experimental goal of finding X-ray periodicity due to modulation of the coma by a rotating, asymmetric nucleus, we were extraordinarily lucky in our time of observation and choice of target: the solar wind was relatively stable over the time of our observations (Fig. 4c). K. Dennerl (1998, private communication) reported statistically significant variations in the X-ray emission morphology from comet C/Tabur 1996 Q1, as observed by ROSAT, on length scales of $\sim 10^{3}-10^{4} \mathrm{~km}$. 


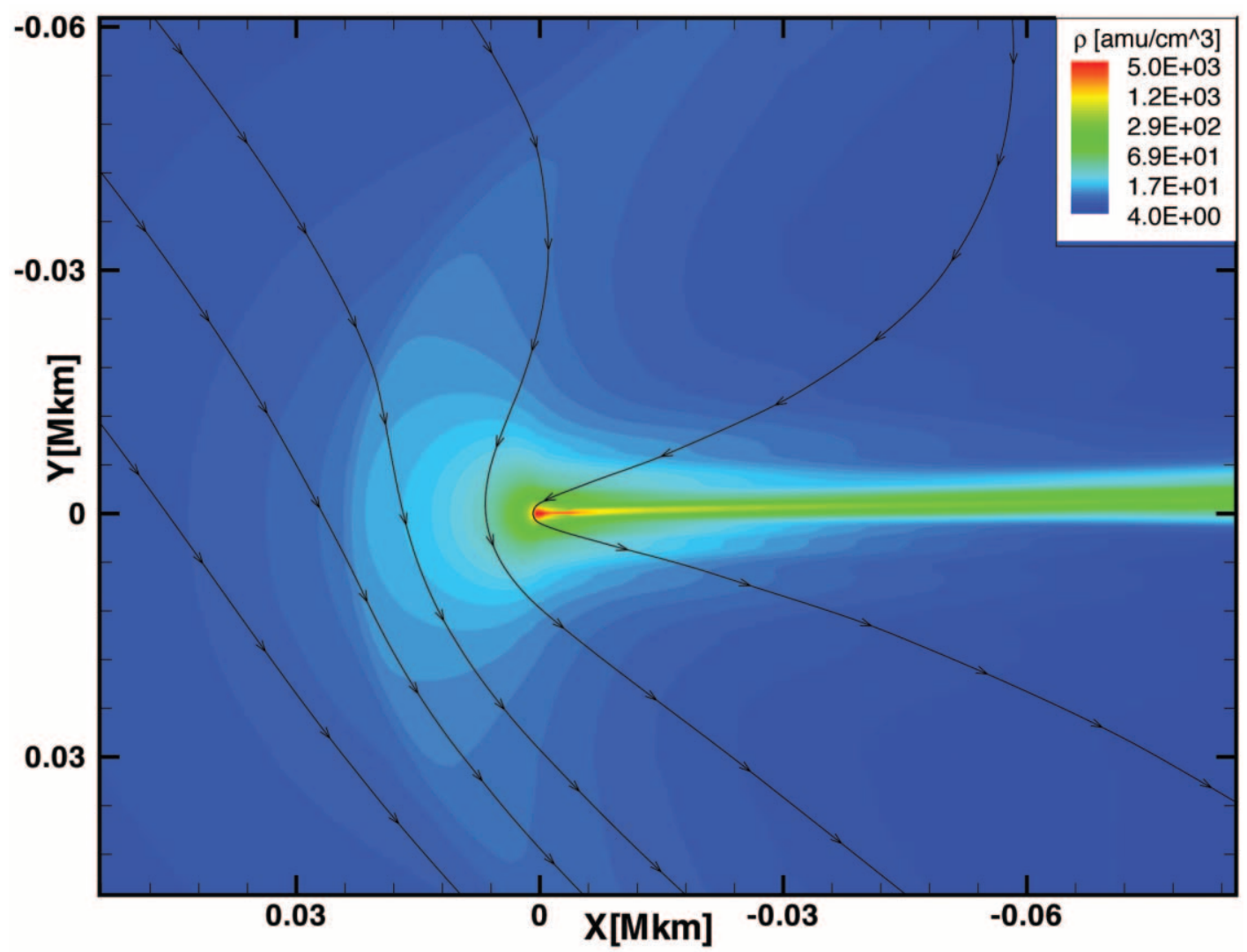

FIG. $5 b$

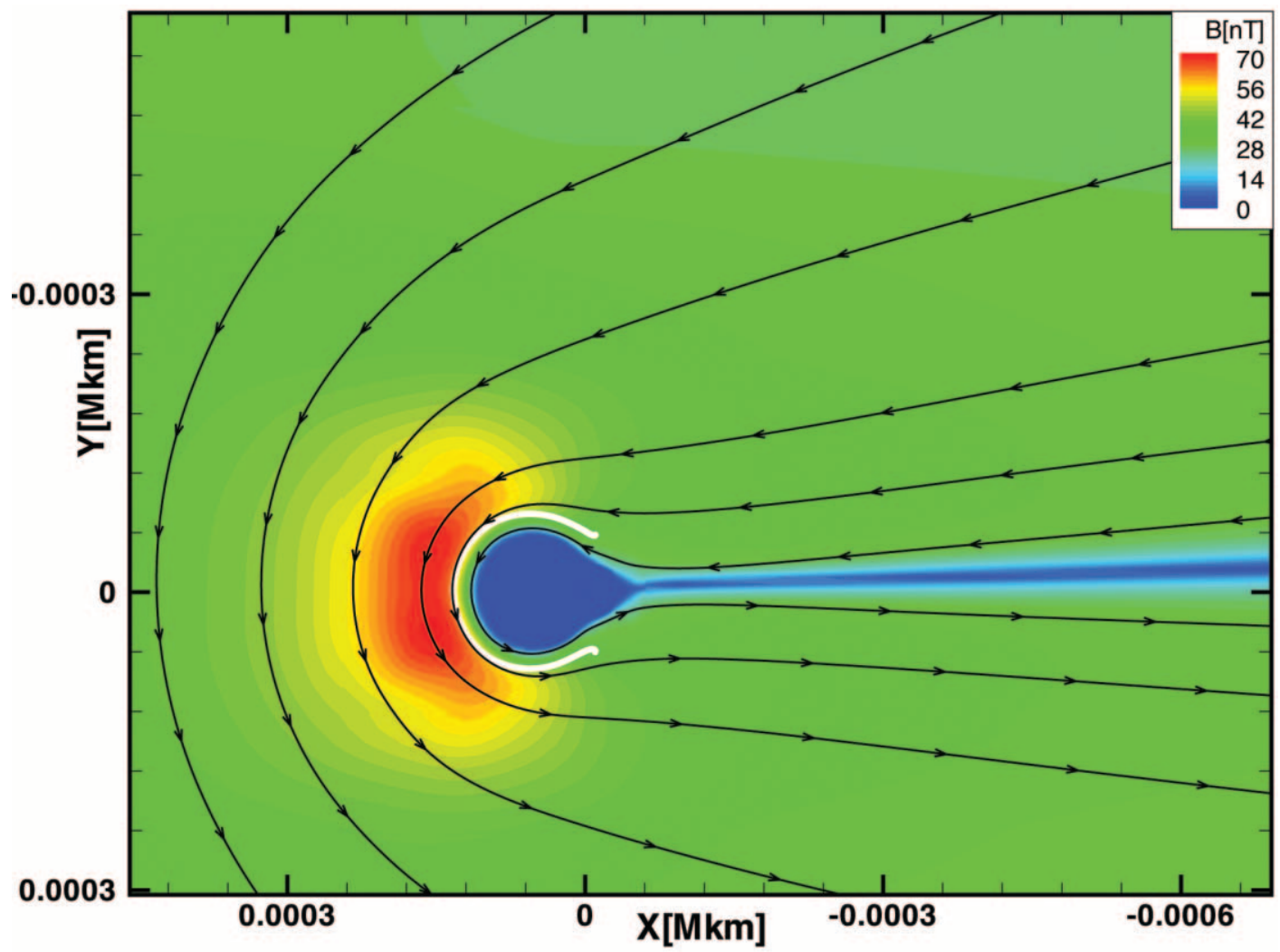

Fig. $5 c$ 

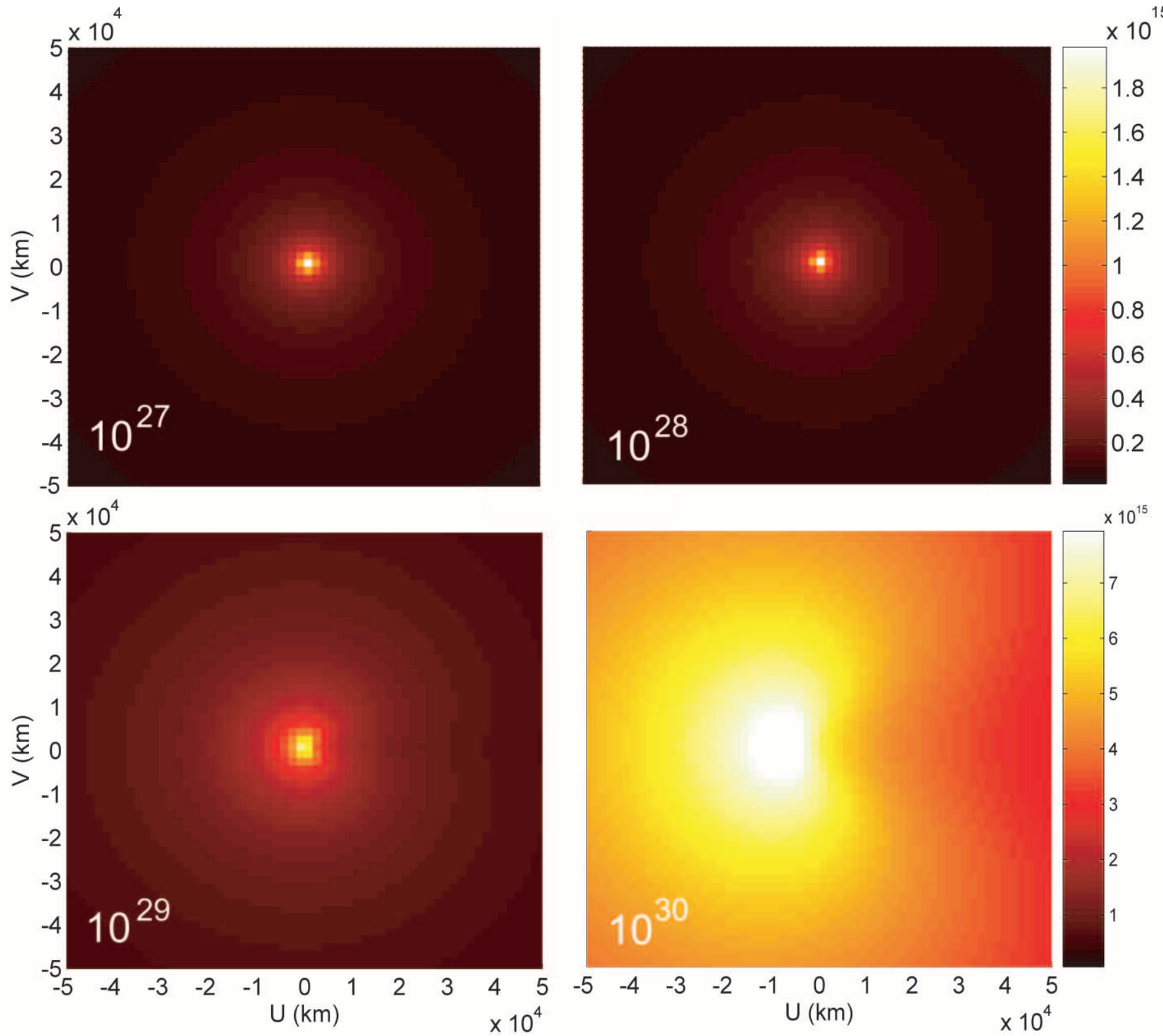

FIG. $6 a$

Fig. 6.-Predicted morphology for the X-ray emission from comet Encke. (a) Morphology as a function of comet gas production rate, following Bodewits et al. (2004). Note the decreasing concentration of model source function and the increasing importance of diffuse halo emission in the extended coma as the gas production rate increases. (b) Model neutral gas density distributions for the dominant neutral species, water and its dissociation products, in the coma of Encke. (c) Predicted oxygen and carbon charge state distribution in the coma of Encke along the comet-Sun axis from the Bodewits model, using the $A C E$ initial charge state distribution given in Table 4.

Images of Tabur's coma taken hours apart show knots of emission lighting up in different locations at different times. Small regions of apparent enhanced brightness a few arcseconds in extent were also found using Chandra for C/1999 S4 (LINEAR) and C/McNaught-Hartley (2001), but were not statistically significant above the $2 \sigma$ level. Because of the compact nature of the emission region, the time for crossing of the coma by gas emitted from the nucleus is on the order of $12 \mathrm{hr}$, and by the solar wind is on the order of a few minutes.

We find good agreement between our observed X-ray light curve and the newly refined $11.1 \mathrm{hr}$ nucleus rotation period of Fernández et al. (2005; our Fig. 2a). Belton et al. (2005) also find an $11.2 \mathrm{hr}$ best-fit period for the rotation of the comet. In their earlier work, Luu \& Jewitt (1990) also found an 11 or $22 \mathrm{hr}$ periodicity possible but argued for a $15 \mathrm{hr}$ phasing as more likely. From the X-ray data, we can rule out periods shortward of $9 \mathrm{hr}$ and longward of $12 \mathrm{hr}$. Returning to the low-level or "baseline" emission time series for the 1997 EUVE/ROSAT light curve of Encke, we find peaks in the power spectrum at $\sim 23 \mathrm{hr}$. The power spectrum is noisy, though, and we cannot determine whether an $11 \mathrm{hr}$ period would fit the data, due to the sparseness of the light curve sampling. We can, however, state that the $11.1 \mathrm{hr}$ period here is consistent with the 1997 pre-outburst measurements.

It is possible that our light curve is influenced by fluctuations in the solar wind or incorrectly removed variations in the background signal of the detector. However, examining the background signal in the ACIS-S data, and the SOHO Proton Monitor solar 


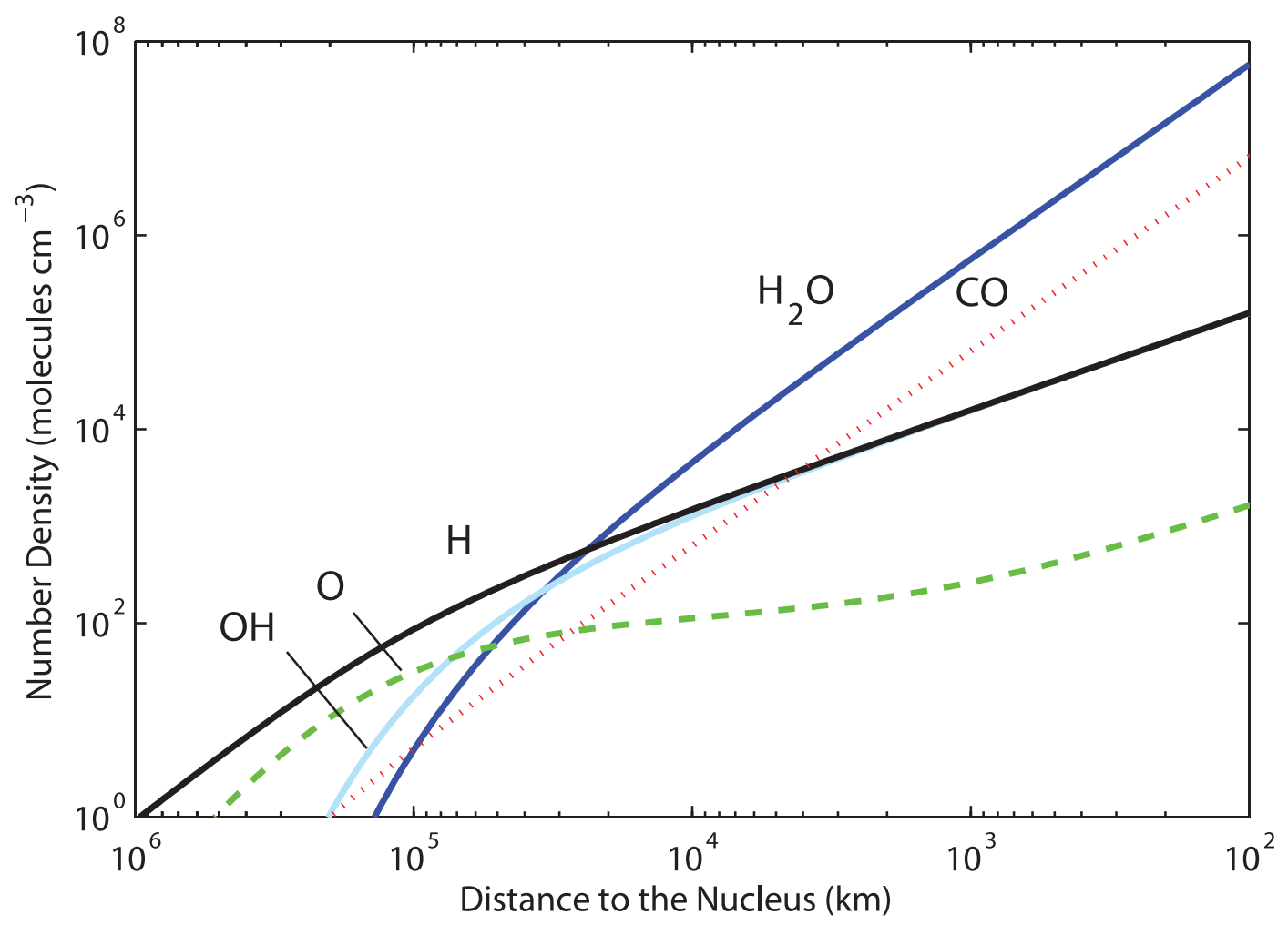

FIG. $6 b$

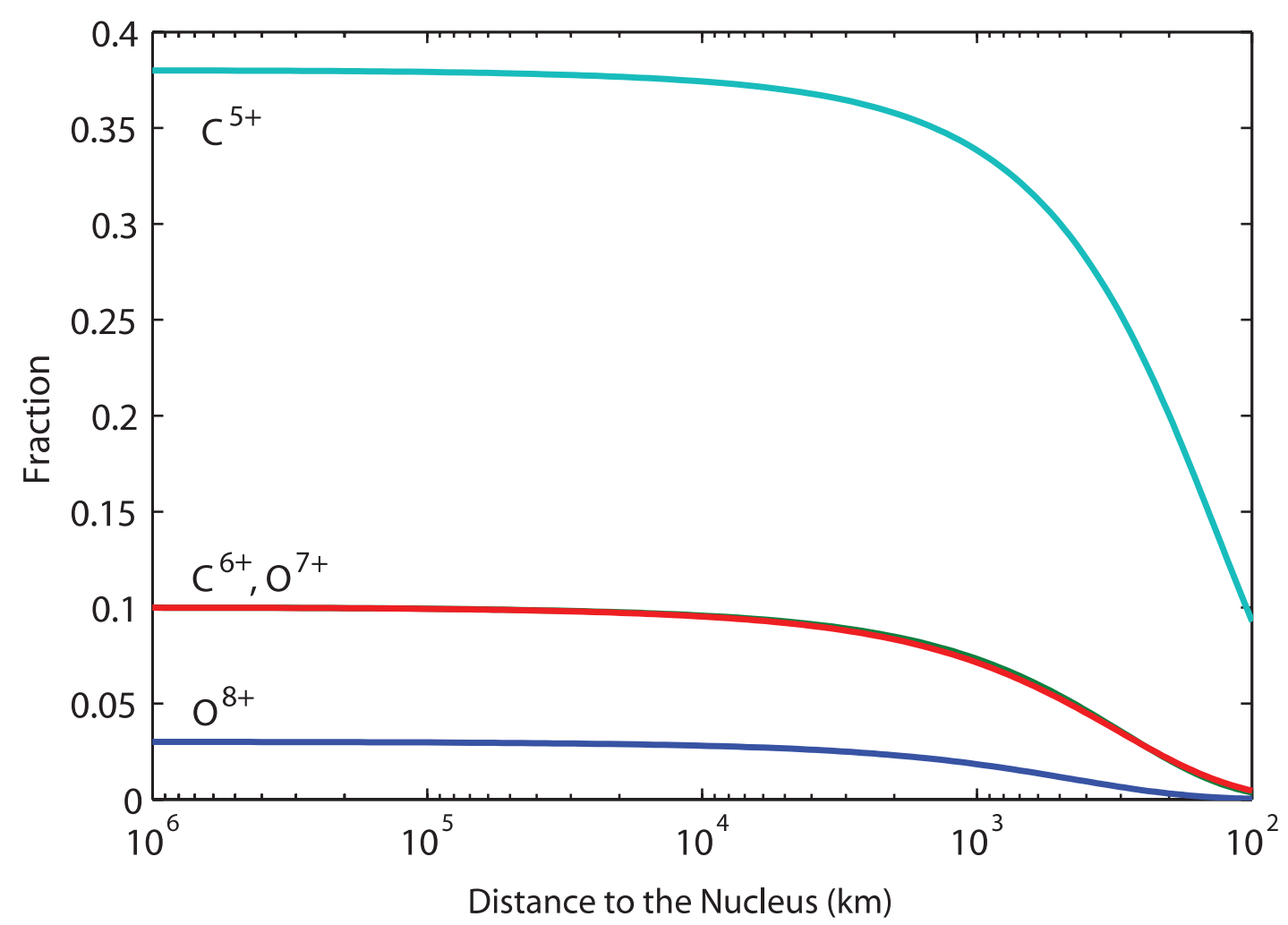

FIG. $6 c$ 
wind proton flux trends over the time share of our observations, we find no trends in the background and only a linear secular decrease in the solar wind flux. The dust and gas emission behavior with time and heliocentric distance have been well documented by Sekanina (1988a, 1988b) and Mäkinen et al. (2001) and shown to have asymmetric, seasonal behavior caused by localized, rotationally variable regions of emission on the surface. Physically, we estimate that for $\sim 1 \mathrm{~km} \mathrm{~s}^{-1}$ gas emission speeds and a core $\mathrm{X}$-ray emission region with greater than $50 \%$ of the total emission in a region less than $18,000 \mathrm{~km}$ in radius, asymmetric emission patterns of gas from the surface of the nucleus can modulate the structure of the comet's atmosphere, and hence its X-ray emission pattern, on timescales of less than onefifth of a day.

Charge state ratios.-We expect softer photons near the nucleus, as the solar wind traverses the coma and its net ionization is reduced; this effect was measured in situ by the Giotto probe during its 1986 flyby of comet 1P/Halley (Fuselier et al. 1991). The most prominent $\mathrm{X}$-ray lines are due to oxygen, so we have studied the $\mathrm{O}$ VII $/ \mathrm{O}$ VIII ratio. (While $\mathrm{O}^{6+}$ is the most abundant oxygen ion in the solar wind, only $\mathrm{O}^{+8}$ and $\mathrm{O}^{+7}$ concern us here, as only $\mathrm{O}$ VIII and $\mathrm{O}$ VII emission, created when $\mathrm{O}^{+8}$ and $\mathrm{O}^{+7}$ charge exchange, fall in the X-ray region of the spectrum.) From our spatial model of the charge state distributions for Encke (Fig. 6c), because of the collisionally thin nature of the system, we do not expect any appreciable variation in the $\mathrm{O}^{+7} / \mathrm{O}^{+8}$ ratio, and hence in the observed $\mathrm{O}$ VII $/ \mathrm{O}_{\mathrm{VIII}}$ line emission, at distances $>1000 \mathrm{~km}$ from the nucleus. Unfortunately, the effective spatial resolution and count rate of the Encke imagery are too low to allow us to determine the spatial dependence of the $\mathrm{O}^{+7} / \mathrm{O}^{+8}$ ratio. A search for variations in the $\mathrm{O}$ VII/O VIII line ratio due to reduction of the solar wind as it traverses the gas coma was also inconclusive for C/1999 S4 (LINEAR), but a clear trend of increasing $\mathrm{O}$ VII $/ \mathrm{O}$ VIII emission toward the nucleus was found in our reanalysis of the Chandra observations of C/McNaughtHartley 2001 (Krasnopolsky et al. 2002). It is not clear whether the difference in the two results is due to overfilling of the S3 chip by C/1999 S4 (LINEAR) in 2000 July or due to variability in the solar wind $\mathrm{O}^{+7} / \mathrm{O}^{+8}$ abundance. Neugebauer et al. (2000) argue that the $\mathrm{O}^{+7} / \mathrm{O}^{+8}$ ratio is highly variable in the solar wind, so that the changing ratio of $\mathrm{O}^{+7} / \mathrm{O}^{+8}$ in the coma of C/McNaught-Hartley 2001 and the relatively constant ratio found in C/1999 S4 (LINEAR) may simply be due to a much smaller initial amount of $\mathrm{O}^{+7}$ in the solar wind impacting the coma of C/McNaught-Hartley 2001. From the Chandra $\mathrm{X}$-ray spectra, the comets appear to be encountering very different $\mathrm{O}^{+7} / \mathrm{O}^{+8}$ solar wind compositions, as evidenced from the $560 \mathrm{eV} / 660 \mathrm{eV}$ line ratios (Fig. 3c).

Charge exchange spectral model.-Several spectral models are now available to simulate the observed X-ray emission. Here we employ the model of Bodewits et al. (2004) to include the properties of both the neutral comet (spatial density, composition) and the ionized solar wind (speed, composition, spatial density) in determining the behavior of the X-ray emission. In this first results paper, the model is used to broadly understand the plethora of new phenomena seen in the 2003 November Chandra observations of Encke and to show that they arise from a common source. In a follow-up paper, a highly detailed spectral analysis will be presented.

As described above, our model promulgates a detailed morphological model of the neutral coma interacting with the planar flow of the incoming solar wind, yielding a two-dimensional $\mathrm{X}$-ray emissivity. A three-dimensional integration assuming cylindrical symmetry around the comet-Sun axis viewed from the
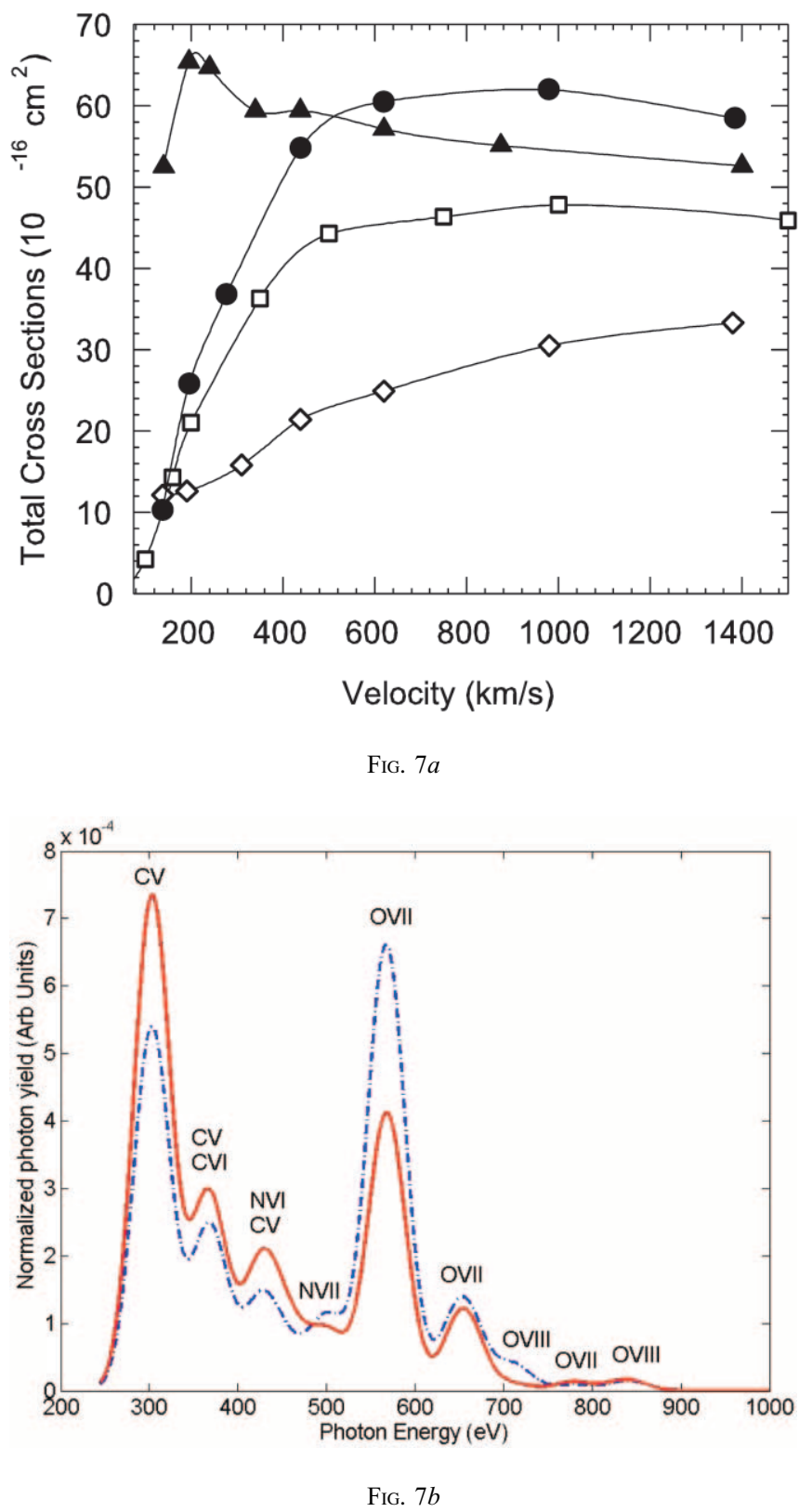

FIG. 7.- (a) Total cross sections for charge exchange of an electron between a highly charged ion and a cometary neutral, as a function of relative ion velocity. Cross sections are given in units of $10^{-16} \mathrm{~cm}^{-2}$, the hard sphere cross section for a hydrogen atom. Filled triangles denote the cross sections for $\mathrm{O}^{+8}$, filled circles the cross sections for $\mathrm{O}^{+7}$, diamonds the cross sections for $\mathrm{C}^{+5}$, and squares the cross sections for $\mathrm{C}^{+6}$. The largest relative change over the range of observed solar wind ion speeds occurs for the $\mathrm{C}^{+6}$ and $\mathrm{O}^{+8}$ ions. (b) Encke X-ray spectra for two different solar wind speeds, assuming a resolution of $60 \mathrm{eV}$, for the collisionally thin case. Blue curve: The $200 \mathrm{~km} \mathrm{~s}^{-1}$ solar wind speed. Red curve: The $600 \mathrm{~km} \mathrm{~s}^{-1}$ solar wind speed. The largest changes in the spectra occur near $350,500,650$, and $820 \mathrm{eV}$.

observer's phase angle in turn yields the absolute intensity of cometary emission lines (Fig. 6a). Effects of the Chandra instrument response were simply modeled by assuming a spectrometer resolution of $60 \mathrm{eV}$ and the Cycle 5 effective areas (Chandra Observer's Manual, Ver. 4.0). To arrive at a spatially dependent spectrum, cascade spectra have to be calculated for all the ions included in the model. Care has been exercised in using velocitydependent, state-selective electron capture cross sections for atomic hydrogen from Fritsch \& Lin (1984) and Shipsey et al. (1983). Forbidden lines are included, assuming a triplet/singlet ratio of 
$3: 1$. Multiple electron capture, which tends to increase Ly $\alpha$ lines, is not included in the model. To get absolute intensities, a solar wind heavy-ion flux of $1.70 \times 10^{5}$ ions $\mathrm{cm}^{-2} \mathrm{~s}^{-1}$ was assumed.

The as-observed line intensities from the best-fit XSPEC eight emission line fit to the data are presented in Figures $3 a$ and $3 b$. Using solar wind velocities of $600 \mathrm{~km} \mathrm{~s}^{-1}$ (the measured velocity during the Encke observations from $A C E$ data) and $200 \mathrm{~km} \mathrm{~s}^{-1}$ (typical of the slow, thick solar wind at low heliographic latitudes/ in the ecliptic), velocity-dependent charge exchange cross sections (Fig. 7a), and charge state ratios for the fast and slow solar winds from Schwadron \& Cravens (2000), from first principles we have simulated the emission spectra (Fig. $7 b$ ). It is immediately clear that the Encke spectra are most similar to the $600 \mathrm{~km} \mathrm{~s}^{-1}$ model, consistent with the $A C E$ and $S O H O$ solar wind speed and temperature measurements. The dominant cause of the highly elevated $\mathrm{C} / \mathrm{O}$ line ratios is due to the highly elevated cross section for $\mathrm{C}^{+6}$ charge exchange at $600 \mathrm{~km} \mathrm{~s}^{-1}$. The high particle speed directly affects the $380 \mathrm{eV} \mathrm{C/560} \mathrm{eV} \mathrm{O} \mathrm{VII}$ line ratio in the Chandra spectrum, as does the unusual charge state, elevating the importance of the carbon lines (Fig. $3 c$ ). The initial population of excited states $(n l)$ following charge exchange is strongly dependent on the collision velocity. The solar wind densities as determined by $A C E$ are also in good qualitative agreement with the solar wind state as deduced from the Encke X-ray spectrum.

New lines. - Because the Encke-solar wind system was collisionally thin to charge exchange at $Q_{\text {gas }}<10^{28} \mathrm{~mol} \mathrm{~s}^{-1}$, the spectrum may allow for new line detections. That is, for oxygen the collisionally thin spectrum is not "filled in" by all possible emission in the $\mathrm{O}$ VII and $\mathrm{O}$ VIII lines, while the less abundant species with higher cross sections for charge exchange are still near their maximum possible luminosity (Fig. 6c). Emission due to $\mathrm{Ne}$ and $\mathrm{Fe}$, which are both abundant in the solar wind, should be found at $826 \mathrm{eV}$ (Fe XVII), $880 \mathrm{eV}$ (Fe XVIII), and $940 \mathrm{eV}$ ( Ne IX). While there is an apparent peak at $826 \mathrm{eV}$ in our Chandra spectrum (Fig. 3), we cannot claim a definitive detection of an Fe line, as there is a stronger $\mathrm{O}^{+8}$ line at $796 \mathrm{eV}$ and there is no obvious need for two lines in the XSPEC modeling of the data at greater than $3 \sigma$ statistical significance. We do find a $5 \sigma$ line at an energy of $984 \mathrm{eV}$, which we assign to Ne Ix. Ne Ix emission barely fell below the background sensitivity limit for C/1999 S4 (LINEAR) (Lisse et al. 2001) and was detected in C/McNaught-Hartley 2001 by Krasnopolsky et al. (2002) with line center at $\sim 960 \mathrm{eV}$.

Emission due to charge exchange by another abundant solar wind species, $\mathrm{N}^{+6}$, should be present but is very hard to resolve. The Bodewits spectral model demonstrates why it is so hard to detect $\mathrm{N}$ vi versus $\mathrm{C}$ vi: on a per atom basis, the nearby $\mathrm{C}$ vi lines are much stronger than the nitrogen lines. $\mathrm{C}$ VI emission alone would produce a $380 \mathrm{eV} / 450 \mathrm{eV}$ line ratio of 4.0 , as opposed to the observed ratio of $3.0 \pm 0.6(2 \sigma)$. While highly interesting, we can at most claim this as a tentative detection of $\mathrm{N}$ VI emission, worthy of further study but not yet conclusive. A similar conclusion was found by P. Beiersdorfer (2003, private communication) in fitting the Chandra C/1999 S4 (LINEAR) X-ray spectra to laboratory electron-ion trap measurements of charge exchange emission by helium-like and hydrogen-like $\mathrm{C}, \mathrm{N}$, and $\mathrm{O}$.

\section{CONCLUSIONS}

Comet Encke was observed on 2003 November 24 for 15 continuous hours using the Chandra ACIS-S imaging spectrometer. Compared to observations of other comets, the Chandra results were unusual:

1. The coma of the comet was clearly collisionally thin to charge exchange with the solar wind up to the central pixel containing the nucleus. The "typical" crescent-shaped emission region was not found. Instead, X-ray emission from comet Encke was resolved on scales of 500-40,000 km, with unusual morphology due to the presence of a low-density, collisionally thin (to charge exchange) coma.

2. A light curve with a peak-to-peak amplitude of $20 \%$ and a period $10.5 \pm 1.5(2 \sigma) \mathrm{hr}$ was found over the $15 \mathrm{hr}$ observing period, consistent with recent evaluations of the nuclear rotation period of $\sim 11 \mathrm{hr}$ (Fernández et al. 2005; Belton et al. 2005). Without a collisionally thin coma, modulation of the X-ray signal by variable nuclear gas emission would not affect the charge exchange interaction, except over the largest scales where the effects are moderated and diluted.

3. A spectrum dominated by charge exchange emission from solar wind $\mathrm{C}, \mathrm{O}, \mathrm{Ne}$, and possibly $\mathrm{N}$ ions was obtained. The measured and inferred charge state of the solar wind for this low-latitude comet was unusually "cold." Very unusual oxygen and carbon line ratios were seen in the 200-700 eV range. There was a marked absence of impulsive variations of the X-ray emission and changes in the solar wind flux during the observations, and the solar wind speed was very high, $\sim 600 \mathrm{~km} \mathrm{~s}^{-1}$.

These findings are consistent with the moderate emission rate of neutral gas from Encke $\left(\sim 7 \times 10^{27} \mathrm{~mol} \mathrm{~s}^{-1}\right)$ and contemporaneous measurements of the solar wind made by $\mathrm{SOHO}$ and $A C E$ showing dominance of the inner heliosphere by a postshock bubble of expanding solar wind plasma containing material from throughout the corona, mixing the behavior seen in high and low heliographic latitude solar wind flow.

The SOHO CELIAS heavy-ion and MTOF Proton Monitor data were graciously provided by the University of Maryland $S O H O$ project, http://umtof.umd.edu/pm. The SOHO SEM solar X-ray monitor data are used courtesy of H. Ogawa at the University of Southern California, http://www.usc.edu/dept/space_science/semdata.htm. The Encke gas production rates were derived by T. Mäkinen from SOHO SWAN observations, and the Encke gas and dust coma images were supplied courtesy of D. Schleicher and L. Woodney. The $A C E$ solar wind heavy-ion data were obtained courtesy of T. Zurbuchen and J. Raines of the University of Michigan and of the $A C E$ SWICS team, http://www.srl.caltech.edu/ACE. We are grateful for the cometary ephemerides of D. K. Yeomans et al. found at http://ssd.jpl.nasa.gov/horizons.html used to reduce our data. Help with the MHD modeling was provided by Y. Jia at the University of Michigan. C. Lisse was supported in part by SMAO observing grants NAG GO45167X, and D. Bodewits and R. Hoekstra would like to acknowledge support within the FOM-EURATOM association agreement.

\section{REFERENCES}

Arnaud, K. A. 1996, in ASP Conf. Ser. 101, Astronomical Data Analysis Software and Systems V, ed. G. H. Jacoby \& J. Barnes (San Francisco: ASP), 17 Beiersdorfer, P., et al. 2003, Science, 300, 1558

Belton, M., et al. 2005, Icarus, 175, 181

Bodewits, D., Juhasz, Z., Tielens, A. G. G. M., \& Hoekstra, R. 2004, ApJ, 606, L81

Cravens, T. E. 1997, Geophys Res. Lett., 24, 105

2002, Science, 296, 1042

Dennerl, K., et al. 1997, Science, 277, 1625

Dryer, M. 1998, AIAA J., 36, 365

Dryer, M., et al. 2001, Sol. Phys., 204, 265

2004, Space Weather, 2, 9001 
Fernández, Y. R., et al. 2005, Icarus, 175, 194

Festou, M., \& Barale, O. 2000, AJ, 119, 3119

Flammer, K. 1991, in Comets in the Post-Halley Era, ed. R. L. Newburn, M. Neugebauer, \& J. Rahe (Dordrecht: Kluwer), 1125

Fritsch, W., \& Lin, C. D. 1984, J. Phys. B, 17, 3271

Fry, C., et al. 2001, J. Geophys. Res., 106, 20985 2003, J. Geophys. Res. 108(A2), 1070

2004, IEEE Trans. Plasma Phys., 32(4), 1489

Fuselier, S., et al. 1991, ApJ, 379, 734

Gombosi, T., et al. 1996, J. Geophys. Res., 101, 15233

Häberli, R., et al. 1997, Icarus, 130, 373

Kharchenko, V., \& Dalgarno, A. 2000, J. Geophys. Res., 105, 18351 2001, ApJ, 554, L99

Kharchenko, V., et al. 2003, ApJ, 585, L73

Krasnopolsky, V., et al. 2002, Icarus, 160, 437

Lisse, C. M., \& Christian, D. J. 2002, in ASP Conf. Ser. 264, Continuing the Challenge of EUV Astronomy: Current Analysis and Prospects for the Future, ed. S. B. Howell, J. Dupuis, D. Golombek, F. M. Walter, \& J. Cullison (San Francisco: ASP), 254
Lisse, C. M., Cravens, T. E., \& Dennerl, K. 2004, in Comets II, ed. M. C. Festou, H. A. Weaver, \& H. U. Keller (Tucson: Univ. Arizona Press), 631 Lisse, C. M., et al. 1996, Science 274, 205 1999, Icarus, 141, 316 2001, Science, 292, 1343

Luu, J., \& Jewitt, D. 1990, Icarus, 86, 69 Mäkinen, T., et al. 2001, Icarus, 152, 268

McComas, D., et al. 1998, Geophys. Res. Lett., 25, 1

Mukai, T., et al. 1987, A\&A, 187, 129

Neugebauer, M., et al. 1987, A\&A, 187, 21 2000, J. Geophys. Res., 105, 20949

Reme, H., et al. 1987, A\&A, 187, 33

Schwadron, N., \& Cravens, T. E. 2000, ApJ, 544, 558

Sekanina, Z. 1988a, AJ, 95, 911

. 1988b, AJ, 96, 1455

Shipsey, E., et al. 1983, Phys. Rev. A, 27, 821

Wegmann, R., \& Dennerl, K. 2005, A\&A, 430, L33

Wegmann, R., Dennerl, K., \& Lisse, C. M. 2004, A\&A, 428, 647 